\title{
An Efficient Synthesis of Highly Functionalized [5,6] Aromatic Spiroketals by Hetero-Diels-Alder Reaction
}

State Key Laboratory of Applied Organic Chemistry, College of Chemistry and Chemical Engineering, Lanzhou University, Lanzhou, 730000, P. R. China Guanglian Zhou, Jianrong Zhu, Zhixiang Xie*, and Ying Li* xiezx@1zu.edu.cn; liying@1zu.edu.cn

\section{Supporting Information}

\section{List of contents}

General Information........................................ 1

Experimental Procedures and Data...........................S2

${ }^{1} \mathrm{H}$ and ${ }^{13} \mathrm{C}$ NMR Spectra of Products .................................. 13

References.............................................S50

\section{General information}

All moisture-sensitive reactions were performed under an atmosphere of Ar. Glassware was flame dried prior to use. THF, $\mathrm{Et}_{2} \mathrm{O}$ and benzene were dried by distillation over $\mathrm{Na} / \mathrm{K} \mathrm{CH}_{2} \mathrm{Cl}_{2}$ was dried by distillation over $\mathrm{NaH}$. Unless otherwise stated, solvents and reagents were used as received. Column chromatography was generally performed on silica gel (200-300 mesh) and TLC inspections were on silica gel GF254 plates. NMR spectra were recorded at $400 \mathrm{MHz} / 100 \mathrm{MHz}\left({ }^{1} \mathrm{H} \mathrm{NMR} /{ }^{13} \mathrm{C}\right.$ NMR $)$ or 300 $\mathrm{MHz} / 75 \mathrm{MHz}\left({ }^{1} \mathrm{H} \mathrm{NMR} /{ }^{13} \mathrm{C} \mathrm{NMR}\right)$ at $21^{\circ} \mathrm{C}$ in $\mathrm{CDCl}_{3}$. Chemical shifts $(\delta)$ are reported as follows: chemical shift, multiplicity $(\mathrm{s}=$ singlet, $\mathrm{d}=$ doublet, $\mathrm{t}=$ triplet, $\mathrm{dd}=$ doublet of doublet, $\mathrm{dt}=\mathrm{doublet}$ of triplet, $\mathrm{b}=$ broad). IR spectra were recorded on a Nicolet 670 FTIR spectrophotometer and reported in wavenumbers $\left(\mathrm{cm}^{-1}\right)$. Mass spectral (MS) data were obtained on a V.G.ZAB-HS mass spectrometer. High-resolution mass spectra (HRMS) were obtained on a Bruker Daltonics APEX II 47e mass spectrometer. 


\section{Experimental Procedures and Data}<smiles>CC(=O)OCc1ccc(OC(C)=O)c(OC(C)=O)c1</smiles>

5-Acetoxy-2-acetoxymethylphenol (12a). To a stirred solution of 2,4-diacetoxybenzaldehyde (21) ${ }^{1}$ $(2.22 \mathrm{~g}, 10 \mathrm{mmol})$ in dry THF (50 ml) was slowly added a $2 \mathrm{M}$ THF solution of borane-DMS complex $(5.0 \mathrm{ml}, 10 \mathrm{mmol})$ at $0{ }^{\circ} \mathrm{C}$ under nitrogen. The reaction was stirred for one hour at room temperature and was then quenched at $0{ }^{\circ} \mathrm{C}$ with water $(2 \mathrm{ml})$. The mixture was evaporated to dryness under reduced pressure to give a crude product which was purified by flash silica gel chromatography $(70: 30$ P.E. : EtOAc). 12a was obtained as a white wax $1.98 \mathrm{~g}(89 \%)$. IR (KBr) 3392, 1736, 1610, 1432, 1373, 1213, $1150 \mathrm{~cm}^{-1} ;{ }^{1} \mathrm{H}$ NMR $\left(300 \mathrm{MHz}, \mathrm{CDCl}_{3}\right) \delta 2.10(\mathrm{~s}, 3 \mathrm{H}), 2.28(\mathrm{~s}, 3 \mathrm{H}), 5.07$ (s, 2H), $6.65(\mathrm{~d}, 1 \mathrm{H}, J=7.5$ $\mathrm{Hz}), 6.66(\mathrm{~s}, 1 \mathrm{H}), 7.25(\mathrm{~d}, 1 \mathrm{H}, J=7.8 \mathrm{~Hz}), 8.15(\mathrm{~s}, 1 \mathrm{H}) ;{ }^{13} \mathrm{C} \mathrm{NMR}\left(100 \mathrm{MHz}, \mathrm{CDCl}_{3}\right) \delta$ 20.79, 21.04, 62.48, 110.58, 113.52, 119.56, 132.39, 152.43, 156.32, 169.39, 173.66. MS (EI) $m / z(\%): 224\left(\mathrm{M}^{+}, 100\right)$, 182 (62), $164(36), 122(41)$.

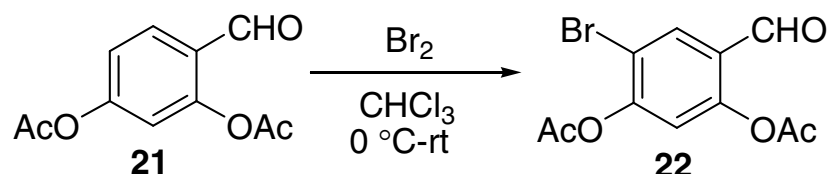

5-Bromo-2,4-diacetoxybenzaldehyde (22). $1.6 \mathrm{~g}$ of bromine $(10 \mathrm{mmol})$ were added to a solution, maintained at $0{ }^{\circ} \mathrm{C}$, of $2.22 \mathrm{~g}(10 \mathrm{mmol})$ of 2,4-diacetoxybenzaldehyde (21) in $20 \mathrm{ml}$ of chloroform. The reaction mixture was allowed to warm slowly to room temperature. After being stirred for 6 hours at the same temperature, the mixture was neutralized with $100 \mathrm{ml}$ of a saturated aqueous sodium bicarbonate, washed with $100 \mathrm{ml}$ of a saturated aqueous sodium chloride solution, dried over anhydrous magnesium sulphate and concentrated to dryness under reduced pressure. The crude product was recrystallized in $\mathrm{CH}_{2} \mathrm{Cl}_{2}$ to yield 22 2.14g (71\%) as a pink solid, Mp 90-91 ${ }^{\circ} \mathrm{C}$. IR (KBr) 1778, 1701, 1369, 1188, 1137 , $1025 \mathrm{~cm}^{-1} ;{ }^{1} \mathrm{H} \mathrm{NMR}\left(300 \mathrm{MHz}, \mathrm{CDCl}_{3}\right) \delta 2.38(\mathrm{~s}, 3 \mathrm{H}), 2.39(\mathrm{~s}, 3 \mathrm{H}), 7.13(\mathrm{~s}, 1 \mathrm{H}), 8.12(\mathrm{~s}, 1 \mathrm{H}), 10.03(\mathrm{~s}$, $1 \mathrm{H}) ;{ }^{13} \mathrm{CNMR}\left(75 \mathrm{MHz}, \mathrm{CDCl}_{3}\right) \delta 20.76,113.96,119.28,126.83,134.86,151.17,152.93,167.41$, 168.40, 186.27; MS (EI), m/z (\%): $300\left(\mathrm{M}^{+}, 0.12\right), 302$ (0.12), 258 (2.1), 260 (2.0), 216 (12.6), 218 (12), $43(100)$. 
<smiles>CC(=O)Oc1cc(OC(C)=O)c(C=O)cc1Br</smiles>

22

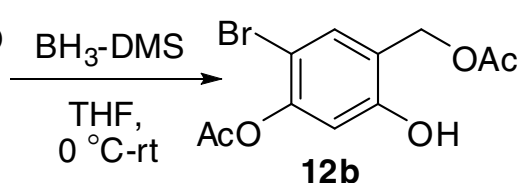

$12 b$

5-Acetoxy-4-bromo-2-methyleneacetoxyphenol (12b). 12b was similarly synthesized as a white wax in $86 \%$ yield. IR (KBr) 3400, 1769, 1739, 1411, 1376, 1228, 1198, $1166 \mathrm{~cm}^{-1} ;{ }^{1} \mathrm{H}$ NMR (300 MHz, $\left.\mathrm{CDCl}_{3}\right) \delta 2.12(\mathrm{~s}, 3 \mathrm{H}), 2.34(\mathrm{~s}, 3 \mathrm{H}), 5.03(\mathrm{~s}, 2 \mathrm{H}), 6.70(\mathrm{~s}, 1 \mathrm{H}), 7.48(\mathrm{~s}, 1 \mathrm{H}), 8.13(\mathrm{~s}, 1 \mathrm{H}) ;{ }^{13} \mathrm{C}$ NMR $(75$ $\left.\mathrm{MHz}, \mathrm{CDCl}_{3}\right) \delta 20.70,20.78,61.73,105.91,112.94,121.63,135.39,149.52,155.66,168.52,173.43 ;$ MS (EI) m/z (\%): $302\left(\mathrm{M}^{+}, 12\right), 304\left(\mathrm{M}^{+}, 12\right), 260$ (25), 262 (24), 242 (6), 244 (6), 200 (100), 202 (100), $172(21), 174(20), 163(18), 93(22)$.<smiles>Cc1ccc2c(c1)OCC2=O</smiles>

$8 a$

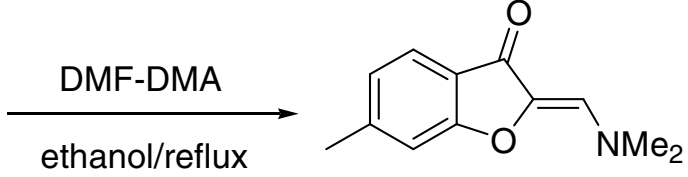

$9 a$

(E)-2-Dimethylaminomethylene-6-methyl-3(2H)-Benzofuranone (9a). 6-Methyl-3(2H)-Benzofuranone (8a) $1.48 \mathrm{~g}$ (10 mmol) was dissolved in absolute ethanol (20 ml); dimethylformamide diethylacetal $1.76 \mathrm{~g}(12 \mathrm{mmol})$ in ethanol $(6 \mathrm{ml})$ was added dropwise to the refluxing mixture. After 4 hours under reflux, the cooled mixture was evaporated leaving a red solid which was washed with diethyl ether and then chromatographed on a silica column ( $3: 2$ dichloromethane : acetone), yield 9a $1.66 \mathrm{~g}(82 \%)$ as a pale-yellow solid, Mp 165-166 ${ }^{\circ} \mathrm{C}$. IR (KBr) 1675, 1575, $1106 \mathrm{~cm}^{-1} ;{ }^{1} \mathrm{H}$ NMR $\left(300 \mathrm{MHz}, \mathrm{CDCl}_{3}\right) \delta 2.42$ (s, 3H), $3.24(\mathrm{~s}, 6 \mathrm{H}), 6.92(\mathrm{~d}, 1 \mathrm{H}, J=7.8 \mathrm{~Hz}), 6.97(\mathrm{~s}, 1 \mathrm{H}), 6.99(\mathrm{~s}, 1 \mathrm{H}), 7.64(\mathrm{~d}, 1 \mathrm{H}, J=7.8 \mathrm{~Hz}) ;{ }^{13} \mathrm{C}$ NMR $\left(75 \mathrm{MHz}, \mathrm{CDCl}_{3}\right) \delta 22.94,112.07,121.30,122.54,123.08,129.70,130.56,144.21,162.06$, 179.59; MS (EI) $m / z(\%): 203\left(\mathrm{M}^{+}, 100\right), 188$ (45), 161 (37), 77 (8).

3(2H)-Benzofuranones $9 \mathbf{b}$ and $9 \mathbf{c}$ were similarly synthesized.<smiles>CN=C1Oc2ccccc2C1=O</smiles>

$9 b$

(E)-2-Dimethylaminomethylene-3(2H)-Benzofuranone (9b). The product was obtained as a pale-yellow solid in $92 \%$ yield, Mp 112-113 (lit. ${ }^{2} \mathrm{Mp} 114{ }^{\circ} \mathrm{C}$ ). IR (KBr) $1674,1566 \mathrm{~cm}^{-1} ;{ }^{1} \mathrm{H}$ NMR (400 
$\left.\mathrm{MHz}^{\mathrm{CDCl}} \mathrm{CD}_{3}\right) \delta .25(\mathrm{~s}, 6 \mathrm{H}), 7.04(\mathrm{~s}, 1 \mathrm{H}), 7.11(\mathrm{t}, 1 \mathrm{H}, J=8.0 \mathrm{~Hz}), 7.18(\mathrm{~d}, 1 \mathrm{H}, J=8.0 \mathrm{~Hz}), 7.47(\mathrm{t}, 1 \mathrm{H}, J$ $=8.0 \mathrm{~Hz}), 7.77(\mathrm{~d}, 1 \mathrm{H}, J=8.0 \mathrm{~Hz}) ;{ }^{13} \mathrm{C} \mathrm{NMR}\left(100 \mathrm{MHz}, \mathrm{CDCl}_{3}\right) \delta 112.16,121.89,123.34,123.94$, 129.79, 131.51, 133.08, 161.65, 179.97; MS (EI) m/z (\%): $189\left(\mathrm{M}^{+}, 100\right), 174(39), 160(11), 147(25)$, $91(8), 77(4)$.<smiles>CN/C=C1\Oc2cc(OC)ccc2C1=O</smiles>

9c

(E)-2-Dimethylaminomethylene-6-methoxyl-3(2H)-Benzofuranone (9c). The product was obtained as a pale-yellow solid in $86 \%$ yield, Mp $154-155^{\circ} \mathrm{C}$. IR (KBr) 1672, $1098 \mathrm{~cm}^{-1} ;{ }^{1} \mathrm{H}$ NMR $(400 \mathrm{MHz}$, $\left.\mathrm{CDCl}_{3}\right) \delta 3.25(\mathrm{~s}, 6 \mathrm{H}), 3.80(\mathrm{~s}, 3 \mathrm{H}), 6.60(\mathrm{~s}, 1 \mathrm{H}), 6.67(\mathrm{~d}, 1 \mathrm{H}, J=8.0 \mathrm{~Hz}), 6.86(\mathrm{~s}, 1 \mathrm{H}), 7.62(\mathrm{~d}, 1 \mathrm{H}, J=$ 8.0 Hz); ${ }^{13} \mathrm{C} \mathrm{NMR}\left(100 \mathrm{MHz}, \mathrm{CDCl}_{3}\right) \delta 55.55,95.71,110.90,117.06,124.09,129.91,130.19,163.81$, 164.61, 179.33; MS (EI) m/z (\%): 219 (M+, 100), 204 (49), 203 (33), 177 (22), 161 (20), 149 (19), 129 (24), 71 (29).

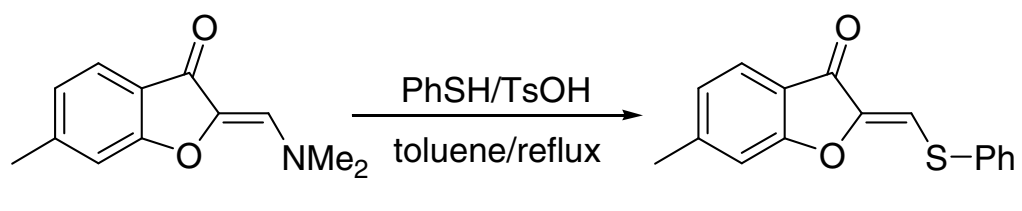

$9 a$

$10 \mathrm{a}$

(E)-2-Phenylthiomethylene-6-methyl-3(2H)-Benzofuranone (10a). A stirred mixture of $(E)$-2-dimethylaminomethylene-6-methyl-3(2H)-Benzofuranone (9a) $609 \mathrm{mg}$ (3 mmol), p-toluenesulfonic acid monohydrate $0.95 \mathrm{~g}(2.5 \mathrm{mmol})$, and benzenethiol $0.363 \mathrm{mg}(3.3 \mathrm{mmol})$ in toluene $15 \mathrm{ml}$ was heated at reflux under nitrogen for 6 hours until 9a disappeared on TLC. The solvent was removed in vacuo, and the residue was purified by flash chromatogeaphy (16: 1 P.E. : EtOAc) to afford 10a as a yellow solid $716 \mathrm{mg}(89 \%), \mathrm{Mp} 123-124{ }^{\circ} \mathrm{C} . \mathrm{IR}(\mathrm{KBr}) 1686,1630,1574,1434,1286,1130 \mathrm{~cm}^{-1} ;{ }^{1} \mathrm{H} \mathrm{NMR}(300 \mathrm{MHz}$, $\left.\mathrm{CDCl}_{3}\right) \delta 2.45(\mathrm{~s}, 3 \mathrm{H}), 6.98(\mathrm{~d}, 1 \mathrm{H}, J=7.8 \mathrm{~Hz}), 7.05(\mathrm{~s}, 1 \mathrm{H}), 7.12(\mathrm{~s}, 1 \mathrm{H})$ 7.36-7.38 (m, 3H), 7.51-7.52 $(\mathrm{m}, 2 \mathrm{H},) ; 7.62(\mathrm{~d}, 1 \mathrm{H}, J=7.8 \mathrm{~Hz}) ;{ }^{13} \mathrm{C} \mathrm{NMR}\left(75 \mathrm{MHz}, \mathrm{CDCl}_{3}\right) \delta 22.57,112.93,116.63,119.89,124.17$, 124.69, 128.43, 129.50, 130.67, 132.78, 145.99, 148.65, 165.58, 180.16; MS (EI) m/z (\%): $268\left(\mathrm{M}^{+}\right.$, 100), 235 (12), 191 (14), 135 (34), 134 (89), 77 (18). 
Thioethers $\mathbf{1 0 b}$ and 10c were similarly synthesized.<smiles>O=C1/C(=C/Sc2ccccc2)Oc2ccccc21</smiles>

$10 \mathrm{~b}$

(E)-2-Phenylthiomethylene-3(2H)-Benzofuranone (10b). The product was obtained as a yellow solid in $92 \%$ yield, $\mathrm{Mp} 82-83{ }^{\circ} \mathrm{C}$. IR (KBr) 1682, 1633, 1575, 1465, 1298, 1189, $1118 \mathrm{~cm}^{-1} ;{ }^{1} \mathrm{H}$ NMR (400 $\left.\mathrm{MHz}, \mathrm{CDCl}_{3}\right) \delta 7.20(\mathrm{~m}, 3 \mathrm{H}), 7.40(\mathrm{~m}, 3 \mathrm{H}), 7.60(\mathrm{~m}, 3 \mathrm{H}), 7.73(\mathrm{~d}, 1 \mathrm{H}, J=8.4 \mathrm{~Hz}) ;{ }^{13} \mathrm{C}$ NMR $(100 \mathrm{MHz}$, $\left.\mathrm{CDCl}_{3}\right) \delta 112.78,117.50,122.20,123.22,124.49,128.48,129.50,130.63,132.61,136.48,145.50$, 164.93, 180.38; MS (EI) $m / z(\%): 254\left(\mathrm{M}^{+}, 100\right), 134$ (78), 121 (30), 77 (10).

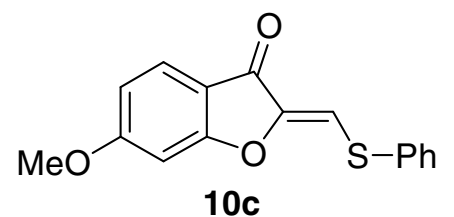

(E)-2-Phenylthiomethylene-6-methoxy-3(2H)-Benzofuranone (10c). The product was obtained as a pale-yellow solid in $93 \%$ yield, Mp 114-116 ${ }^{\circ} \mathrm{C}$. IR (KBr) 1688, 1631, 1591, 1437, 1286, $1123 \mathrm{~cm}^{-1} ;{ }^{1} \mathrm{H}$ NMR (300 MHz, $\left.\mathrm{CDCl}_{3}\right) \delta 3.89(\mathrm{~s}, 3 \mathrm{H}), 6.73(\mathrm{~s}, 1 \mathrm{H}), 6.75(\mathrm{~d}, 1 \mathrm{H} J=8.1 \mathrm{~Hz}), 7.08(\mathrm{~s}, 1 \mathrm{H}), 7.38(\mathrm{~d}, 3 \mathrm{H}$, $J=8.1 \mathrm{~Hz}), 7.53(\mathrm{dd}, 2 \mathrm{H}, J=8.1,2.4 \mathrm{~Hz}), 7.67(\mathrm{~d}, 1 \mathrm{H}, J=8.7 \mathrm{~Hz}) ;{ }^{13} \mathrm{C} \mathrm{NMR}\left(100 \mathrm{MHz}, \mathrm{CDCl}_{3}\right) \delta$ $55.98,96.57,111.89,112.12,115.52,125.73,128.41,129.54,130.72,132.95,146.60,167.25,167.53$, 179.09; MS (EI) m/z (\%): $284\left(\mathrm{M}^{+}, 100\right), 219$ (75), 151 (50), 134 (6), 71 (50), 69 (44), 57 (57).

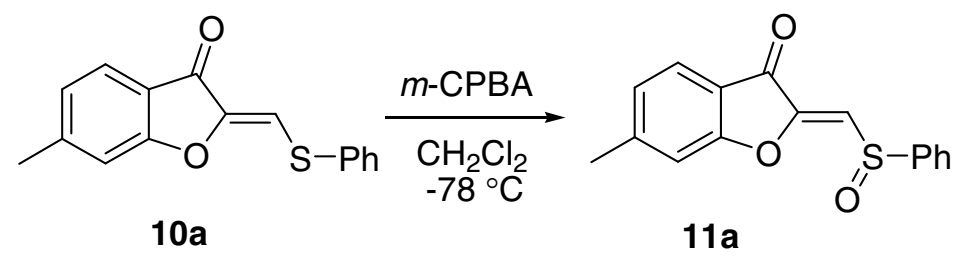

(E)-6-Methyl-2-phenylthiomethylene-3(2H)-Benzofuranone (11a). To a solution of (E)-2-phenylthiomethylene-6-methyl-3(2H)-Benzofuranone (10a) $268 \mathrm{mg}(1 \mathrm{mmol})$ in $\mathrm{CH}_{2} \mathrm{Cl}_{2} 1.5 \mathrm{~mL}$ was added dropwise a solution of $m$-chloroperbenzoic acid $215 \mathrm{mg}$ of $80 \%$ mixture $(1 \mathrm{mmol})$ in $\mathrm{CH}_{2} \mathrm{Cl}_{2}(1 \mathrm{~mL})$ at $-78^{\circ} \mathrm{C}$. The reaction mixture was allowed to warm slowly to $0{ }^{\circ} \mathrm{C}$. After 2 hours TLC analysis $(2: 1$ P.E. : EtOAc) showed loss of starting material. The reaction mixture was partitioned between $\mathrm{CH}_{2} \mathrm{Cl}_{2}(50 \mathrm{ml})$ and $10 \%$ aqueous potassium carbonate solution $(20 \mathrm{ml})$. The organic phase was washed twice with $10 \%$ 
aqueous potassium carbonate solution ( $2 \mathrm{ml}$ ), dried over $\mathrm{MgSO}_{4}$, and purified by flash chromatography (4 : 1 P.E. : EtOAc) to yield $233 \mathrm{mg}(82 \%)$ of sulfoxide 11a as a pale-yellow solid. Mp 148-149 ${ }^{\circ} \mathrm{C}$. IR (KBr) 1705, 1599, 1444, 1287, 1155, 1110, $1041 \mathrm{~cm}^{-1} ;{ }^{1} \mathrm{H}$ NMR (300 MHz, $\left.\mathrm{CDCl}_{3}\right) \delta 2.48(\mathrm{~s}, 3 \mathrm{H}), 6.52$ (s, 1H), 6.99(s, 1H), 7.05 (d, 1H, $J=7.8 \mathrm{HZ}), 7.49-7.56(\mathrm{~m}, 3 \mathrm{H}), 7.65(\mathrm{~d}, 1 \mathrm{H}, J=8.1 \mathrm{HZ}), 7.84(\mathrm{~m}, 2 \mathrm{H})$; ${ }^{13} \mathrm{C}$ NMR $\left(100 \mathrm{MHz}, \mathrm{CDCl}_{3}\right) \delta 22.81,113.32,117.72,118.20,124.26,124.88,125.85,129.62,131.54$, 143.56, 149.73, 151.12, 167.20, 182.41; MS (EI) m/z (\%): $284\left(\mathrm{M}^{+}, 10\right), 268$ (100), 243 (29), 232 (16), 134 (98), 77 (12); HRMS (EI): Calcd. for $\mathrm{C}_{16} \mathrm{H}_{13} \mathrm{SO}_{3}{ }^{+} 285.0580$, Found $285.0584\left(\mathrm{M}+\mathrm{H}^{+}\right)$.

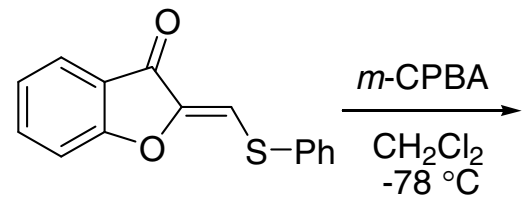

$10 b$<smiles>O=C1/C(=C/S(=O)c2ccccc2)Oc2ccccc21</smiles>

$11 b$

$(E)$, (Z)-2-Phenylthiomethylene-3(2H)-Benzofuranone (11b). To a solution of (E)-2-phenylthiomethylene-3(2H)-Benzofuranone (10b) $254 \mathrm{mg}(1 \mathrm{mmol})$ in $\mathrm{CH}_{2} \mathrm{Cl}_{2} 1.5 \mathrm{~mL}$ was added dropwise a solution of $m$-chloroperbenzoic acid $215 \mathrm{mg}$ of $80 \%$ mixture $(1 \mathrm{mmol})$ in $\mathrm{CH}_{2} \mathrm{Cl}_{2}(1 \mathrm{~mL})$ at $-78{ }^{\circ} \mathrm{C}$. The reaction mixture was allowed to warm slowly to $0{ }^{\circ} \mathrm{C}$. After 2 hours TLC analysis ( $2: 1$ P.E. : EtOAc) showed loss of starting material. The reaction mixture was partitioned between $\mathrm{CH}_{2} \mathrm{Cl}_{2}(50 \mathrm{ml})$ and $10 \%$ aqueous potassium carbonate solution $(20 \mathrm{ml})$. The organic phase was washed twice with $10 \%$ aqueous potassium carbonate solution ( $2 \mathrm{ml}$ ), dried over $\mathrm{MgSO}_{4}$, and purified by flash chromatography (4:1 P.E. : EtOAc) to yield $210 \mathrm{mg}$ (78\%) of sulfoxide 11b $(E: Z=10: 7)$ as a yellow wax. IR (KBr) 1714, 1596, 1464, 1298, 1183, 1098, $1043 \mathrm{~cm}^{-1}$; $(E)$ isomer: ${ }^{1} \mathrm{H}$ NMR $\left(300 \mathrm{MHz}, \mathrm{CDCl}_{3}\right) 6.67(\mathrm{~s}, 1 \mathrm{H})$, 7.22-7.30 (m, 1H), $7.34(\mathrm{~d}, 1 \mathrm{H}, J=8.1 \mathrm{~Hz}) ; 7.50-7.57(\mathrm{~m}, 3 \mathrm{H}) ; 7.70(\mathrm{~d}, 1 \mathrm{H}, J=7.2 \mathrm{~Hz}), 7.66-7.86(\mathrm{~m}$, 2H), 7.83-7.86 (m, 1H); ${ }^{13} \mathrm{C}$ NMR (75 MHz, $\left.\mathrm{CDCl}_{3}\right) \delta 113.15,118.09,120.43,124.14,124.50,125.17$, 129.61, 131.58, 138.45, 143.34, 149.05, 166.58, 182.93; (Z) isomer: ${ }^{1} \mathrm{H}$ NMR $\left(300 \mathrm{MHz}, \mathrm{CDCl}_{3}\right) \delta 6.57$ (s, $1 \mathrm{H}), 7.20(\mathrm{~d}, 1 \mathrm{H}, J=9.0 \mathrm{~Hz}), 7.22-7.30(\mathrm{~m}, 1 \mathrm{H}) ; 7.50-7.57(\mathrm{~m}, 3 \mathrm{H}) ; 7.66-7.77(\mathrm{~m}, 3 \mathrm{H}), 7.83-7.86(\mathrm{~m}$, $1 \mathrm{H}) ;{ }^{13} \mathrm{C}$ NMR (75 MHz, $\left.\mathrm{CDCl}_{3}\right) \delta 113.04,118.09,120.90,124.24,125.11,125.66,129.41,131.43$, 138.39, 144.99, 148.81, 166.80, 182.93; MS (EI) m/z (\%): 270 (M+ $\mathrm{M}^{+}$4), 254 (26), 221 (100), 134 (25), 121 (22), 77 (23); HRMS (EI): Calcd. for $\mathrm{C}_{15} \mathrm{H}_{11} \mathrm{SO}_{3}{ }^{+} 271.0423$, Found $271.0425\left(\mathrm{M}+\mathrm{H}^{+}\right)$. 


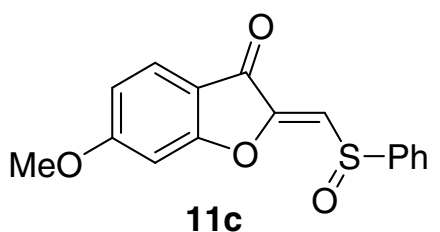

(E)-6-Methoxyl-2-phenylthiomethylene-3(2H)-Benzofuranone (11c). The product was similarly synthesized as a pale-yellow solid in $92 \%$ yield, Mp 128-129 ${ }^{\circ} \mathrm{C}$. IR (KBr) 1708, 1603, 1442, 1291 , 1148, $1102 \mathrm{~cm}^{-1} ;{ }^{1} \mathrm{H}$ NMR (400 MHz, $\left.\mathrm{CDCl}_{3}\right) \delta 3.91(\mathrm{~s}, 3 \mathrm{H}), 6.48(\mathrm{~s}, 1 \mathrm{H}), 6.61(\mathrm{~d}, 1 \mathrm{H}, J=2.4 \mathrm{~Hz}), 6.77$ $(\mathrm{d}, 1 \mathrm{H}, J=8.4 \mathrm{~Hz}), 7.51(\mathrm{~m}, 3 \mathrm{H}), 7.69(\mathrm{~d}, 1 \mathrm{H}, J=8.4 \mathrm{~Hz}), 7.83(\mathrm{~m}, 2 \mathrm{H}) ;{ }^{13} \mathrm{C} \mathrm{NMR}\left(100 \mathrm{MHz}, \mathrm{CDCl}_{3}\right) \delta$ $56.25,97.06,113.01,113.63,117.04,124.14,126.43,129.58,131.47,143.66,150.51,168.60,169.19$, 180.75; MS (EI) m/z (\%): 300 (M+, 5), 284 (10), 251 (100), 151 (20), 135 (13), 77 (15); HRMS (EI): Calcd. for $\mathrm{C}_{16} \mathrm{H}_{12} \mathrm{NaSO}_{4}{ }^{+} 323.0349$, Found $323.0345\left(\mathrm{M}+\mathrm{Na}^{+}\right)$.<smiles>Cc1ccc2c(c1)O/C(=C\S(=O)c1ccccc1)C2=O</smiles>

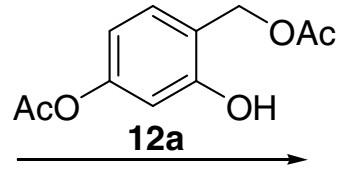

dry $\mathrm{PhH} /$ sealed tube $110{ }^{\circ} \mathrm{C}, 55.2 \%$

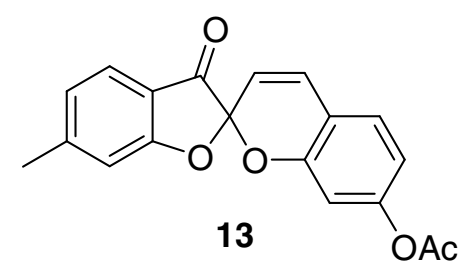

Spiroketal (13). In a sealed tube was stirred 5-acetoxy-2-methyleneacetoxyphenol (12a) $22.4 \mathrm{mg}(0.100$ mmol) and (E)-2-phenylthiomethylene-3(2H)-Benzofuranone (10a) $22.7 \mathrm{mg}(0.08 \mathrm{mmol})$ in benzene $3.0 \mathrm{ml}$ at $110{ }^{\circ} \mathrm{C}$ under argon for 39 hours. After evaporation of excess of reagent under reduced pressure, the brown oil obtained was purified by flash silica gel chromatography (16: 1 P.E. : EtOAc) to give a

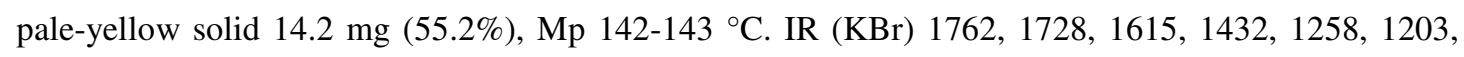
1122, $\mathrm{cm}^{-1} ;{ }^{1} \mathrm{H}$ NMR (300 MHz, $\left.\mathrm{CDCl}_{3}\right) \delta 2.28(\mathrm{~s}, 3 \mathrm{H}), \delta 2.44(\mathrm{~s}, 3 \mathrm{H}), 5.68(\mathrm{~d}, 1 \mathrm{H}, J=10.2 \mathrm{~Hz}), 6.81$ $(\mathrm{m}, 3 \mathrm{H}), 6.96(\mathrm{~d}, 1 \mathrm{H}, J=7.5 \mathrm{~Hz}), 7.04(\mathrm{~d}, 1 \mathrm{H}, J=10.2 \mathrm{~Hz}) 7.24,(\mathrm{~d}, 1 \mathrm{H}, J=8.1 \mathrm{~Hz}), 7.61(\mathrm{~d}, 1 \mathrm{H}, J=$ $8.1 \mathrm{~Hz}) ;{ }^{13} \mathrm{C}$ NMR $\left(100 \mathrm{MHz}, \mathrm{CDCl}_{3}\right) \delta 21.06,22.72,101.50,110.27,113.37,114.89,115.74,116.04$, $116.76,124.44,125.21,127.77,129.19,151.36,151.84,151.95,168.86,170.72,194.69 ; \mathrm{MS}(\mathrm{EI}) \mathrm{m} / \mathrm{z}$ (\%): $322\left(\mathrm{M}^{+}, 37\right), 280$ (25), 251 (100), 237 (27), 77 (29); HRMS (EI): Calcd for $\mathrm{C}_{19} \mathrm{H}_{15} \mathrm{O}_{5}{ }^{+} 323.0914$, Found $323.0919\left(\mathrm{M}+\mathrm{H}^{+}\right)$. 


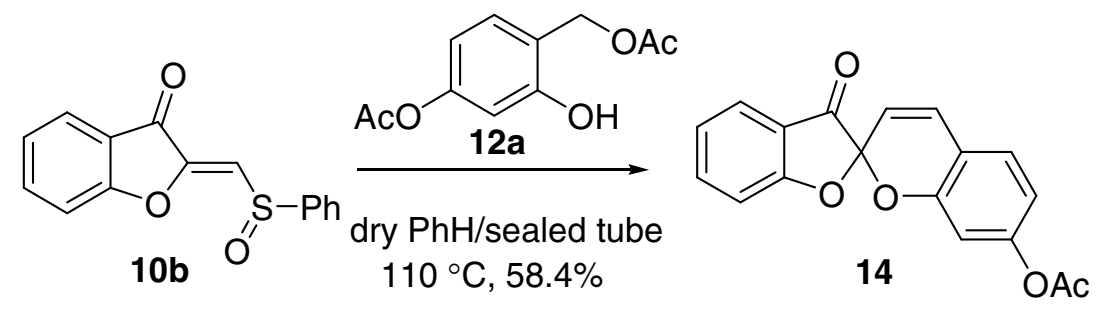

Spiroketal (14). In a sealed tube was stirred 5-acetoxy-2-methyleneacetoxyphenol (12a) $22.4 \mathrm{mg}(0.100$ mmol) and (E), (Z)-2-phenylthiomethylene-3(2H)-Benzofuranone (10b) $21.6 \mathrm{mg}(0.080 \mathrm{mmol})$ in benzene $3.0 \mathrm{ml}$ at $110{ }^{\circ} \mathrm{C}$ under argon for 28 hours. After evaporation of the solvent under reduced pressure, the brown oil obtained was purified by flash silica gel chromatography (16: 1 P.E. : EtOAc) to give 14 as a pale-yellow solid $14.4 \mathrm{mg}(58.4 \%)$, Mp 130-131 ${ }^{\circ} \mathrm{C}$. IR (KBr) 1762, 1732, 1612, 1461 , 1236, 1199, 1143, 1125, $\mathrm{cm}^{-1} ;{ }^{1} \mathrm{H}$ NMR $\left(400 \mathrm{MHz}, \mathrm{CDCl}_{3}\right) \delta 2.28(\mathrm{~s}, 3 \mathrm{H}), 5.69(\mathrm{~d}, 1 \mathrm{H}, J=9.6 \mathrm{~Hz}), 6.80$ $(\mathrm{d}, 1 \mathrm{H}, J=2.0 \mathrm{~Hz}), 6.82(\mathrm{dt}, 1 \mathrm{H}, J=8.0,1.2,0.8 \mathrm{~Hz}), 7.02(\mathrm{~d}, 1 \mathrm{H}, J=8.0 \mathrm{~Hz}), 7.05(\mathrm{~d}, 1 \mathrm{H}, J=9.6 \mathrm{~Hz})$, $7.16(\mathrm{dd}, 1 \mathrm{H}, J=7.6,7.2 \mathrm{~Hz}), 7.24(\mathrm{~d}, 1 \mathrm{H}, J=8.4 \mathrm{~Hz}), 7.69(\mathrm{dd}, 1 \mathrm{H}, J=7.2,7.6 \mathrm{~Hz}), 7.74(\mathrm{~d}, 1 \mathrm{H}, J=$ $8.0 \mathrm{~Hz}) ;{ }^{13} \mathrm{C} \mathrm{NMR}\left(100 \mathrm{MHz}, \mathrm{CDCl}_{3}\right) \delta 21.07,101.16,110.31,113.35,114.64,116.14,116.75,118.16$, $122.95,125.58,127.83,129.37,139.49,151.28,151.89,168.88,170.29,195.40 ;$ MS (EI) $m / z(\%): 308$ $\left(\mathrm{M}^{+}, 9\right), 279$ (6), 266 (7), 237 (100), 210 (5), 181 (3), 137 (10), 65 (26); HRMS (EI): Calcd. for $\mathrm{C}_{18} \mathrm{H}_{13} \mathrm{O}_{5}{ }^{+}$309.0757, Found 309.0757 $\left(\mathrm{M}+\mathrm{H}^{+}\right)$.
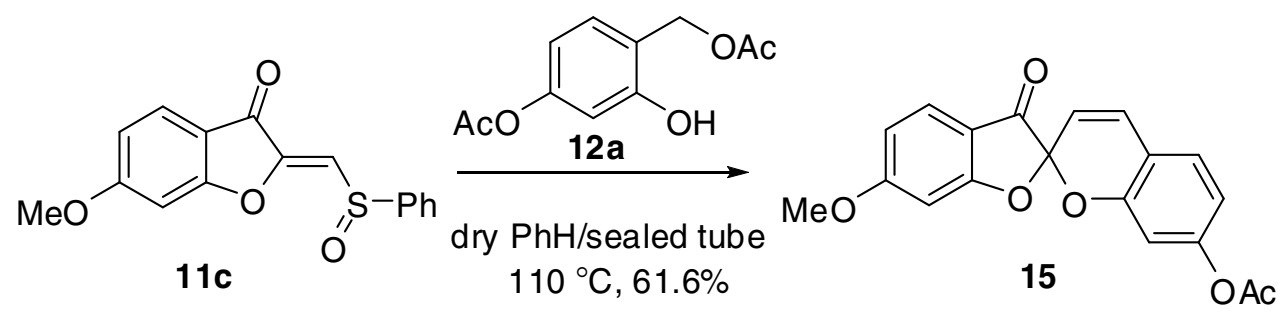

Spiroketal (15). In a sealed tube was stirred 5-acetoxy-2-methyleneacetoxyphenol (12a) $22.4 \mathrm{mg}(0.100$ mmol) and (E)-6-methoxyl-2-phenylthiomethylene-3(2H)-Benzofuranone (11c) $24.2 \mathrm{mg}(0.081 \mathrm{mmol})$ in benzene $3.0 \mathrm{ml}$ at $110{ }^{\circ} \mathrm{C}$ under argon for 39 hours. After evaporation of excess reagent under reduced pressure, the brown oil obtained was purified by flash silica gel chromatography (16: 1 P.E. : EtOAc) to give a pale-yellow solid $16.8 \mathrm{mg}(61.6 \%), \mathrm{Mp} 134-136{ }^{\circ} \mathrm{C} . \mathrm{IR}(\mathrm{KBr}) 1763,1720,1611,1498,1439$, 1284, 1198, 1142, $1121 \mathrm{~cm}^{-1} ;{ }^{1} \mathrm{H}$ NMR (400 MHz, $\left.\mathrm{CDCl}_{3}\right) \delta 2.29(\mathrm{~d}, 3 \mathrm{H}, J=1.8 \mathrm{~Hz}), 3.88(\mathrm{~s}, 3 \mathrm{H}), 5.70$ $(\mathrm{d}, 1 \mathrm{H}, J=10.0 \mathrm{~Hz}), 6.43(\mathrm{~d}, 1 \mathrm{H}, J=2.0 \mathrm{~Hz}), 6.69(\mathrm{dd}, 1 \mathrm{H}, J=8.8,2.4 \mathrm{~Hz}), 6.80(\mathrm{~s}, 1 \mathrm{H}), 6.82,(\mathrm{dd}, 1 \mathrm{H}$, $J=8.0,2.0 \mathrm{~Hz}), 7.05(\mathrm{~d}, 1 \mathrm{H}, J=10.0 \mathrm{~Hz}), 7.24(\mathrm{~d}, 1 \mathrm{H}, J=8.0 \mathrm{~Hz}), 7.64(\mathrm{~d}, 1 \mathrm{H}, J=8.4 \mathrm{~Hz}) ;{ }^{13} \mathrm{C} \mathrm{NMR}$ $\left(100 \mathrm{MHz}, \mathrm{CDCl}_{3}\right) \delta 21.06,56.02,96.42,110.32,111.15,112.30,115.09,116.06,116.83,119.24$, 
<smiles>Cc1ccc2c(c1)O/C(=C\S(=O)c1ccccc1)C2=O</smiles>

$11 a$<smiles>CC(=O)OCc1cc(Br)c(OC(C)=O)cc1O</smiles>

$110^{\circ} \mathrm{C}, 75.6 \%$<smiles>CC(=O)Oc1cc2c(cc1Br)C=CC1(O2)Oc2cc(C)ccc2C1=O</smiles>

Spiroketal (16). In a sealed tube was stirred 5-acetoxy-4-bromo-2-methyleneacetoxyphenol (12b) 30.3 $\mathrm{mg}(0.100 \mathrm{mmol})$ and (E)-6-methyl-2-phenylthiomethylene-3(2H)-Benzofuranone (11a) $22.7 \mathrm{mg}(0.080$ mmol) in benzene $3.0 \mathrm{ml}$ at $110{ }^{\circ} \mathrm{C}$ under argon for 39 hours. After evaporation of excess reagent under reduced pressure, the brown oil obtained was purified by flash silica gel chromatography (16: 1 P.E. : EtOAc) to give a pale-yellow solid 24.2mg (75.6\%). Mp 161-162 ${ }^{\circ} \mathrm{C}$. IR (KBr) 1771, 1728, 1615, 1481, 1426, 1367, 1262, 1193, $1146 \mathrm{~cm}^{-1} ;{ }^{1} \mathrm{H}$ NMR (300 MHz, $\left.\mathrm{CDCl}_{3}\right) \delta 2.35(\mathrm{~s}, 3 \mathrm{H}), \delta 2.45(\mathrm{~s}, 3 \mathrm{H}), 5.73(\mathrm{~d}$, $1 \mathrm{H}, J=10.2 \mathrm{~Hz}), 6.81(\mathrm{~s}, 1 \mathrm{H}), 6.83(\mathrm{~s}, 1 \mathrm{H}), 6.97(\mathrm{~d}, 1 \mathrm{H}, J=6.3 \mathrm{~Hz}), 6.99(\mathrm{~d}, 1 \mathrm{H}, J=9.9 \mathrm{~Hz}), 7.48(\mathrm{~s}$, 1H), $7.60(\mathrm{~d}, 1 \mathrm{H}, J=7.5 \mathrm{~Hz}) ;{ }^{13} \mathrm{C}$ NMR $\left(100 \mathrm{MHz}, \mathrm{CDCl}_{3}\right) \delta 20.72,22.73,101.103,108.99,112.45$, $113.38,115.61,116.31,118.57,124.63,125.26,128.19,130.82,149.01,150.51,152.11,168.03,170.70$, 194.24; MS (EI) m/z (\%): $400\left(\mathrm{M}^{+}, 2\right), 402$ (2), 358 (5), 360 (5), 329 (8), 331 (8), 315 (3), 317 (3), 223 (3) 201 (9), 203(9), 165 (3), 149 (100), 77 (11); HRMS (EI): Calcd. for $\mathrm{C}_{19} \mathrm{H}_{17} \mathrm{BrNO}_{5}{ }^{+}$418.0285, Found $418.0289\left(\mathrm{M}+\mathrm{NH}_{4}^{+}\right)$.<smiles>O=C1/C(=C/S(=O)c2ccccc2)Oc2ccccc21</smiles><smiles>CC(=O)OCc1cc(Br)c(OC(C)=O)c(O)c1O</smiles><smiles></smiles>

Spiroketal (17). In a sealed tube was stirred 5-acetoxy-4-bromo-2-methyleneacetoxyphenol (12b) 30.3 (0.100 mmol) and (E), (Z)-2-phenylthiomethylene-3(2H)-Benzofuranone (11b) $21.1 \mathrm{mg}(0.078 \mathrm{mmol})$ in benzene $3.0 \mathrm{ml}$ at $110{ }^{\circ} \mathrm{C}$ under argon for 39 hours. After evaporation of excess reagent under reduced pressure, the brown oil obtained was purified by flash silica gel chromatography (16: 1 P.E. : EtOAc) to give a pale-yellow solid $27.1 \mathrm{mg}(89.7 \%)$, Mp 132-134 ${ }^{\circ} \mathrm{C}$. IR (KBr) 1771, 1733, 1610, 1480, 1461, 
1242, 1192, $1150 \mathrm{~cm}^{-1} ;{ }^{1} \mathrm{H}$ NMR (300 MHz, $\left.\mathrm{CDCl}_{3}\right) \delta 2.34(\mathrm{~s}, 3 \mathrm{H}), 5.73(\mathrm{~d}, 1 \mathrm{H}, J=10.2 \mathrm{~Hz}), 6.84(\mathrm{~s}$, 1H), $7.00(\mathrm{~d}, 1 \mathrm{H}, J=9.6 \mathrm{~Hz}), 7.02(\mathrm{~d}, 1 \mathrm{H}, J=8.1 \mathrm{~Hz}), 7.16(\mathrm{t}, 1 \mathrm{H}, J=7.5,7.8 \mathrm{~Hz}), 7.49(\mathrm{~s}, 1 \mathrm{H}), 7.66(\mathrm{dt}$, $1 \mathrm{H}, J=1.5,7.2 \mathrm{~Hz}), 7.73(\mathrm{~d}, 1 \mathrm{H}, J=8.4 \mathrm{~Hz}) ;{ }^{13} \mathrm{C} \mathrm{NMR}\left(100 \mathrm{MHz}, \mathrm{CDCl}_{3}\right) \delta 20.75,100.70,109.10$, $112.47,113.33,115.99,117.95,118.51,123.15,125.61,128.35,130.85,139.65,148.99,150.37,168.11$, 170.22, 195.03. MS (EI) m/z (\%): $386\left(\mathrm{M}^{+}, 11\right), 388$ (10), 344 (47), 346 (48), 315 (100), 317 (98), 288 (9), 290 (9), 203 (20), 152 (23), 77 (6); HRMS (EI): Calcd. for $\mathrm{C}_{18} \mathrm{H}_{12} \mathrm{O}_{5}{ }^{+}$386.9863, Found 386.9865 $\left(\mathrm{M}+\mathrm{H}^{+}\right)$.

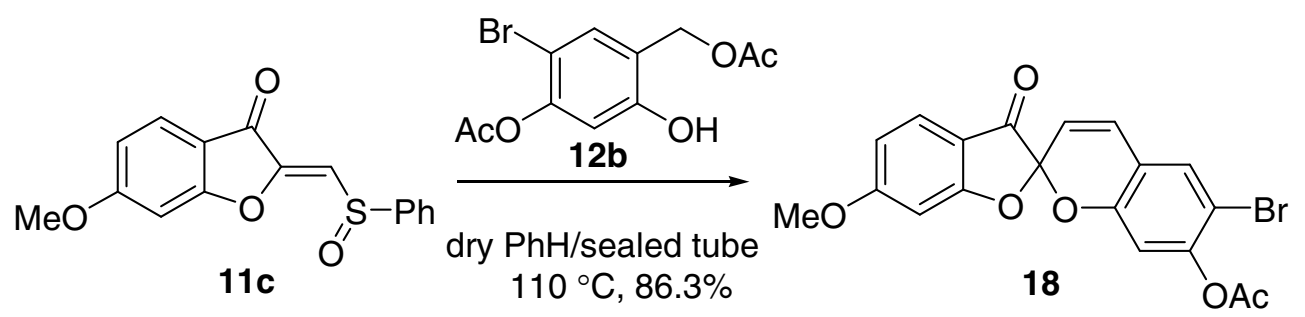

Spiroketal (18). In a sealed tube was stirred 5-acetoxy-4-bromo-2-methyleneacetoxyphenol (12b) 30.3 $\mathrm{mg}(0.100 \mathrm{mmol})$ and $(E)-6$-methoxyl-2-phenylthiomethylene-3(2H)-Benzofuranone (11c) $24.1 \mathrm{mg}$ $(0.080 \mathrm{mmol})$ and in benzene $3.0 \mathrm{ml}$ at $110{ }^{\circ} \mathrm{C}$ under argon for 39 hours. After evaporation of excess reagent under reduced pressure, the brown oil obtained was purified by flash silica gel chromatography (16: 1 P.E. : EtOAc) to give a pale-yellow solid $28.9 \mathrm{mg}(86.3 \%)$. Mp 153-154 ${ }^{\circ} \mathrm{C}$. IR (KBr) 1771,1721 , 1612, 1460, 1372, 1284, 1194, $1148 \mathrm{~cm}^{-1} ;{ }^{1} \mathrm{H}$ NMR (300 MHz, $\left.\mathrm{CDCl}_{3}\right) \delta 2.34$ (s, 3H), 3.89 (s, 3H), 5.75 $(\mathrm{d}, 1 \mathrm{H}, J=9.3 \mathrm{~Hz}), 6.44(\mathrm{~d}, 1 \mathrm{H}, J=2.1 \mathrm{~Hz}), 6.69(\mathrm{dd}, 1 \mathrm{H}, J=8.7,2.4 \mathrm{~Hz}), 6.85(\mathrm{~s}, 1 \mathrm{H}), 6.97(\mathrm{~d}, 1 \mathrm{H}, J=$ $9.3 \mathrm{~Hz}), 7.48,(\mathrm{~s}, 1 \mathrm{H}), 7.64(\mathrm{~d}, 1 \mathrm{H}, J=8.7 \mathrm{~Hz}) ;{ }^{13} \mathrm{C} \mathrm{NMR}\left(100 \mathrm{MHz}, \mathrm{CDCl}_{3}\right) \delta 20.76,56.07,96.32$, $101.73,109.02,110.89,112.52,116.41,118.58,126.84,128.19,130.79,148.93,150.50,168.12,169.64$, 172.75, 192.49; MS (EI) m/z (\%): $416\left(\mathrm{M}^{+}, 21\right), 418$ (21), 374 (98), 376 (100), 345 (92), 347 (93), 315 (52), 317 (53), 134 (82), 71 (92); HRMS (EI): Calcd. for $\mathrm{C}_{19} \mathrm{H}_{17} \mathrm{BrNO}_{6}{ }^{+}$434.0234, Found 434.0231 $\left(\mathrm{M}+\mathrm{NH}_{4}^{+}\right)$.

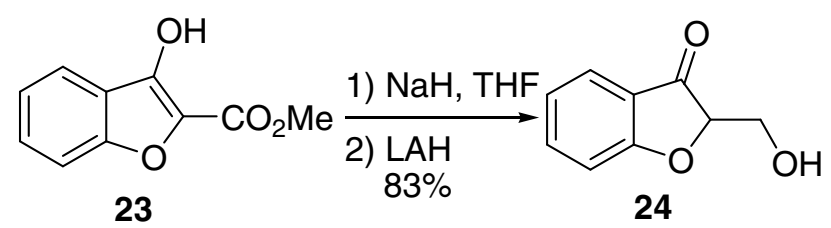

2-Hydroxymethyl-3(2H)-Benzofuranone (24). Sodium hydride $440 \mathrm{mg}$ (11 mmol) was washed with 
$n$-hexane and dried under reduced pressure. Methyl-3-hydroxy-7-methoxybenzofuran-2-carboxylate $(\mathbf{2 3})^{3} 1.92 \mathrm{~g}(10.0 \mathrm{mmol})$ was dissolved in THF $200 \mathrm{ml}$ and the solution was added to the sodium hydide, followed by refluxing the resulting solution for 1 hour. After cooling the solution to $0{ }^{\circ} \mathrm{C}$, aluminum lithium hydride $404 \mathrm{mg}(10.6 \mathrm{mmol})$ was added, and the resulting solution was stirred at $0{ }^{\circ} \mathrm{C}$ for 2 hours. To the reaction solution, a 1:1 mixture $(200 \mathrm{ml})$ of $1 \mathrm{~N}$ hydrochloric acid and saturated brine was added and the organic phase was separated. The aqueous phase was extracted with ethyl acetate. The organic phases were combined and washed with saturated brine, followed by drying over $\mathrm{MgSO}_{4}$. The solvent was evaporated off. The residue was purified by flash chromatography $(2: 1$ P.E. : EtOAc) to yield $1.37 \mathrm{~g}(83 \%)$ of 24 as pallid wax. IR (KBr) 3408, 1712, 1611,1469 $\mathrm{cm}^{-1} ;{ }^{1} \mathrm{H}$ NMR (300 MHz, $\left.\mathrm{CDCl}_{3}\right) \delta 2.32(\mathrm{~b}, 1 \mathrm{H}), 4.08(\mathrm{~m}, 2 \mathrm{H}), 4.66(\mathrm{dd}, 1 \mathrm{H}, J=3.9,4.8 \mathrm{~Hz}), 7.08(\mathrm{dd}, 1 \mathrm{H}, J=7.5,7.8 \mathrm{~Hz}), 7.15$ $(\mathrm{d}, 1 \mathrm{H}, J=8.4 \mathrm{~Hz}), 7.62(\mathrm{~m}, 2 \mathrm{H}) ;{ }^{13} \mathrm{C} \mathrm{NMR}\left(100 \mathrm{MHz}, \mathrm{CDCl}_{3}\right) \delta 61.63,85.97,113.47,121.19,122.02$, 124.11, 138.23, 173.21, 200.38; MS (EI) $m / z(\%): 164\left(\mathrm{M}^{+}, 36\right), 146(100)$.

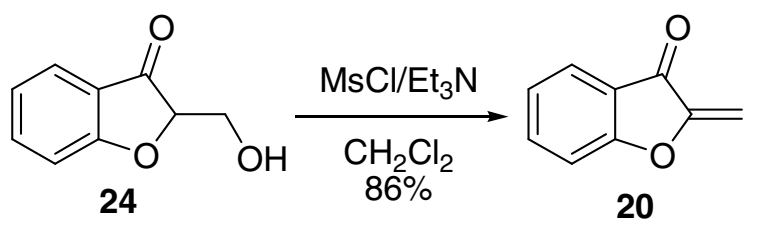

2-Methylene-3(2H)-Benzofuranone (20). 2-Hydroxymethyl-3(2H)-Benzofuranone (24) $328 \mathrm{mg}$ (2 mmol) was dissolved in dry $\mathrm{CH}_{2} \mathrm{Cl}_{2}(10 \mathrm{ml})$ and the solution was cooled to $0{ }^{\circ} \mathrm{C}$. Triethylamine $0.56 \mathrm{~mL}$ (4 mmol) was added quickly, and the reaction mixture was stirred for $10 \mathrm{~min}$. Methanesulfonyl chloride $0.31 \mathrm{ml}(4 \mathrm{mmol})$ was added dropwise, and the resulting solution was stirred at $0{ }^{\circ} \mathrm{C}$ for $30 \mathrm{~min}$. The reaction mixture was diluted with water, and the organic phase was separated. The aqueous phase was extracted with $\mathrm{CH}_{2} \mathrm{Cl}_{2}$. The combined organic phase was washed with $\mathrm{HCl}(1 \mathrm{M}, 1 \mathrm{ml})$ and saturated aqueous $\mathrm{NaHCO}_{3}$ and was dried over $\mathrm{MgSO}_{4}$. After removing the solvent in vacuo, the resulting crude product was purified by flash chromatography (32:1 P.E. : EtOAc) to give $251 \mathrm{mg} 20(86 \%)$ as a white wax. IR (KBr) 1616, $1025 \mathrm{~cm}^{-1} ;{ }^{1} \mathrm{H}$ NMR (300 MHz, CDCl 3$) \delta 5.27$ (d, 1H, J=2.4 Hz), 5.65 (d, 1H, J= $2.4 \mathrm{~Hz}), 7.17(\mathrm{t}, 2 \mathrm{H}, J=6.3,8.4 \mathrm{~Hz}), 7.63(\mathrm{t}, 1 \mathrm{H}, J=7.5,7.8 \mathrm{~Hz}), 7.75(\mathrm{~d}, 1 \mathrm{H}, J=7.5 \mathrm{~Hz}) ;{ }^{13} \mathrm{C} \mathrm{NMR}(75$ $\left.\mathrm{MHz}, \mathrm{CDCl}_{3}\right) \delta 97.25,112.81,122.87,123.25,124.85,137.57,149.06,166.85,182.26$; MS (EI) $\mathrm{m} / z(\%)$ : $146\left(\mathrm{M}^{+}, 9\right), 101(30), 83(44), 59(100)$. 
<smiles>C=C1Oc2ccccc2C1=O</smiles>

20 $\underset{\text { dry } \mathrm{PhH} / \text { sealed tube }}{\mathrm{12a}}$ $110{ }^{\circ} \mathrm{C}, 46.3 \%$

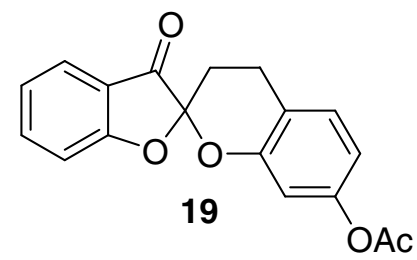

Spiroketal (19). In a sealed tube was stirred 5-acetoxy-2-methyleneacetoxyphenol (12a) $22.4 \mathrm{mg}(0.100$ mmol) and 2-methylene-3(2H)-Benzofuranone (20) $11.7 \mathrm{mg}(0.080 \mathrm{mmol})$ in benzene $3.0 \mathrm{ml}$ at $110{ }^{\circ} \mathrm{C}$ under argon for 28 hours. After evaporation of the solvent under reduced pressure, the brown oil obtained was purified by flash silica gel chromatography (16: 1 P.E. : EtOAc) to give 19 as a pale-yellow solid $11.5 \mathrm{mg}(46.3 \%)$, Mp 132-133 ${ }^{\circ} \mathrm{C}$. IR (KBr) 1763, 1732, 1615, 1200, 1141, $1049 \mathrm{~cm}^{-1} ;{ }^{1} \mathrm{H}$ NMR $\left(300 \mathrm{MHz}, \mathrm{CDCl}_{3}\right) \delta 2.04(\mathrm{dd}, 1 \mathrm{H}, J=14,5.7,2.4 \mathrm{~Hz}), 2.26(\mathrm{~s}, 3 \mathrm{H}), 2.28(\mathrm{~m}, 1 \mathrm{H}), 2.94(\mathrm{dt}, 1 \mathrm{H}, J=16$, 6.0, $2.4 \mathrm{~Hz}), 3.13(\mathrm{ddd}, 1 \mathrm{H}, J=13.0,6.0,3.6 \mathrm{~Hz}), 6.64(\mathrm{~d}, 1 \mathrm{H}, J=1.8 \mathrm{~Hz}), 6.73(\mathrm{dd}, 1 \mathrm{H}, J=8.1,2.4 \mathrm{~Hz})$, $7.06(\mathrm{~d}, 1 \mathrm{H}, J=9.3 \mathrm{~Hz}), 7.13(\mathrm{~m}, 2 \mathrm{H}), 7.66(\mathrm{dt}, 1 \mathrm{H} J=7.8,1.8 \mathrm{~Hz}), 7.73(\mathrm{~d}, 1 \mathrm{H}, J=7.8 \mathrm{~Hz}) ;{ }^{13} \mathrm{C} \mathrm{NMR}$ $\left(100 \mathrm{MHz}, \mathrm{CDCl}_{3}\right) \delta 20.12,21.06,25.36,102.01,110.62,113.54,115.43,118.71,118.88,122.71$, $125.48,129.61,139.12,149.88,152.36,169.32,170.46,195.42 ; \mathrm{MS}(\mathrm{EI}) \mathrm{m} / \mathrm{z}(\%): 310\left(\mathrm{M}^{+}, 33\right), 268$ (60), 239 (22), 180 (22), 147 (36), 137 (100), 121 (39), 84 (90), 77 (20); HRMS (EI): Calcd. for $\mathrm{C}_{18} \mathrm{H}_{18} \mathrm{O}_{5}{ }^{+}$328.1179, Found 328.1186 $\left(\mathrm{M}+\mathrm{NH}_{4}^{+}\right)$. 


\section{${ }^{1} \mathrm{H}$ and ${ }^{13} \mathrm{C}$ NMR Spectra of Products}

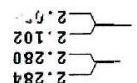

$082^{\circ} z$

$220 \cdot s$

$089 \cdot 97$

$5 S 9 \cdot 9$
659

${ }_{298}^{\tau b z} \cdot 2=$

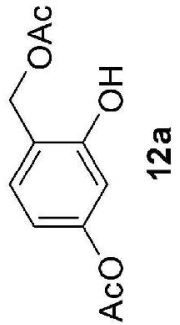

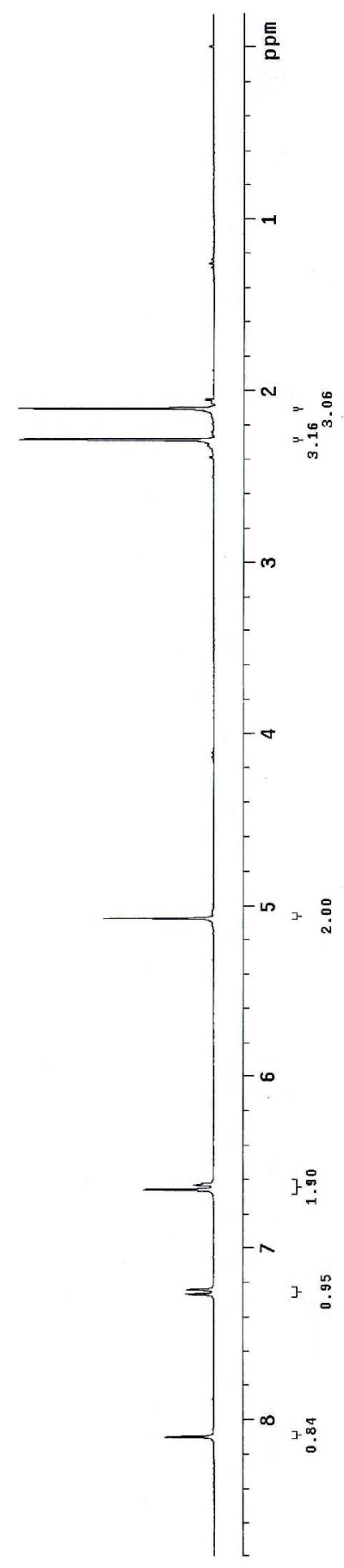




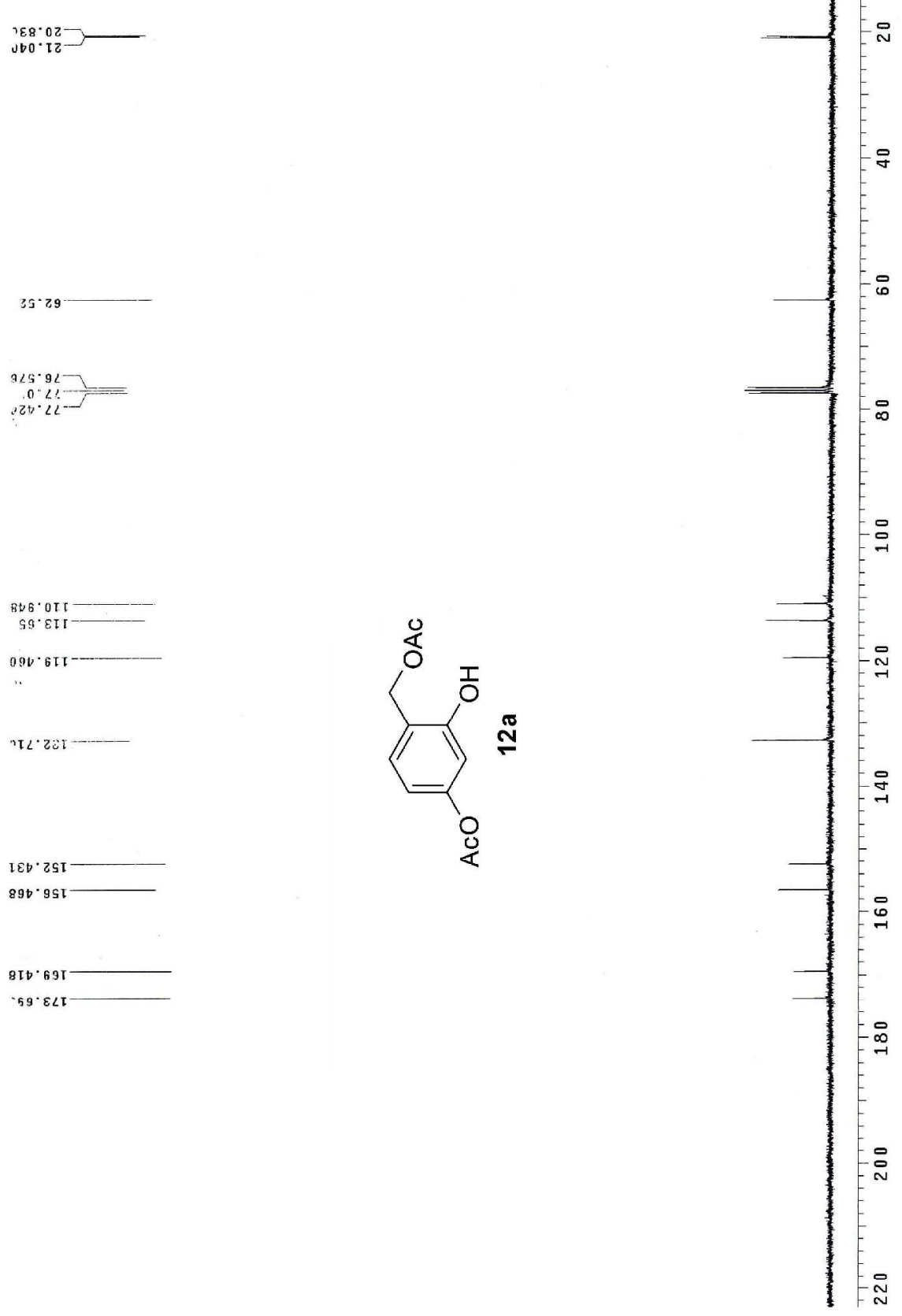




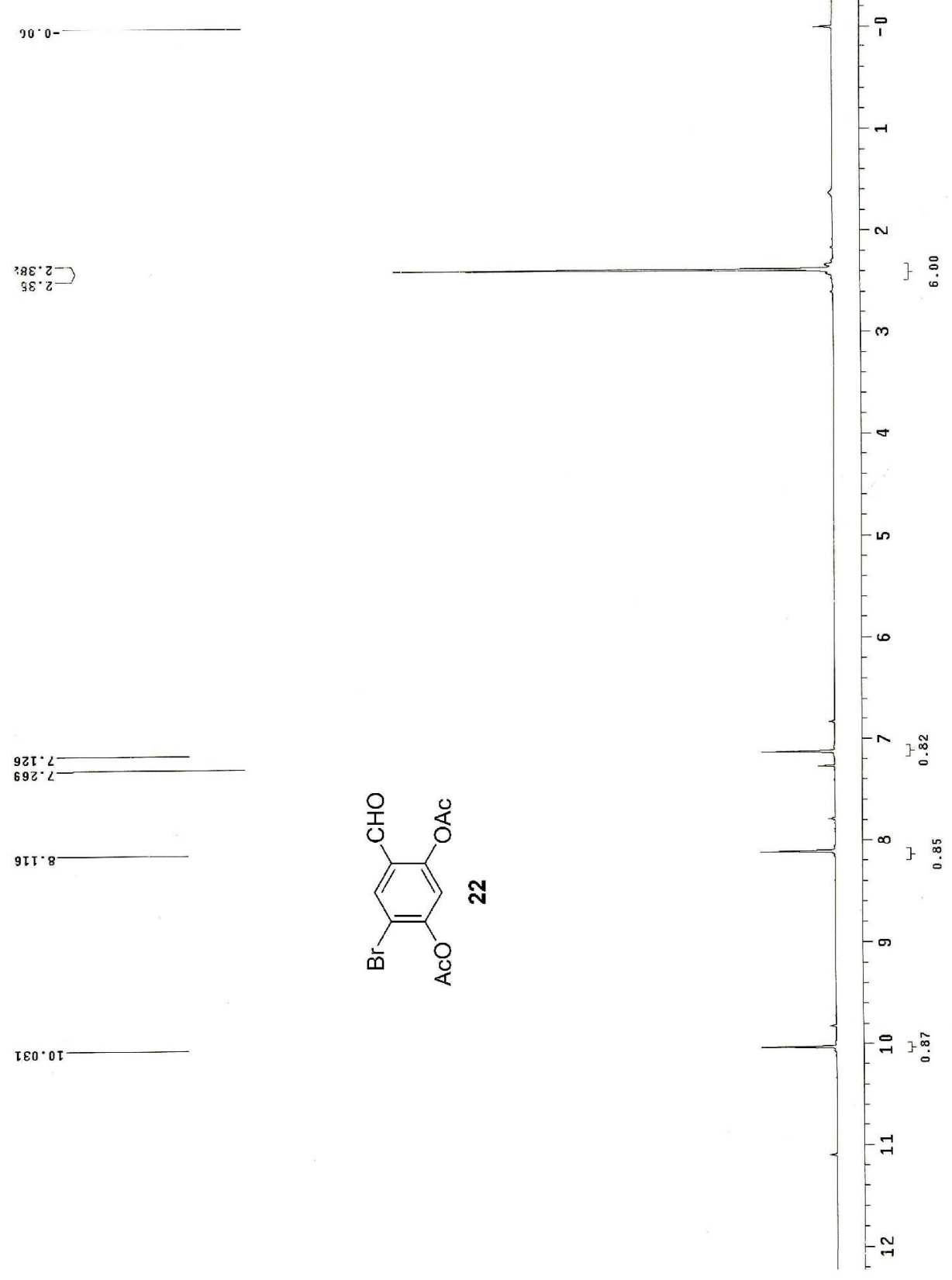



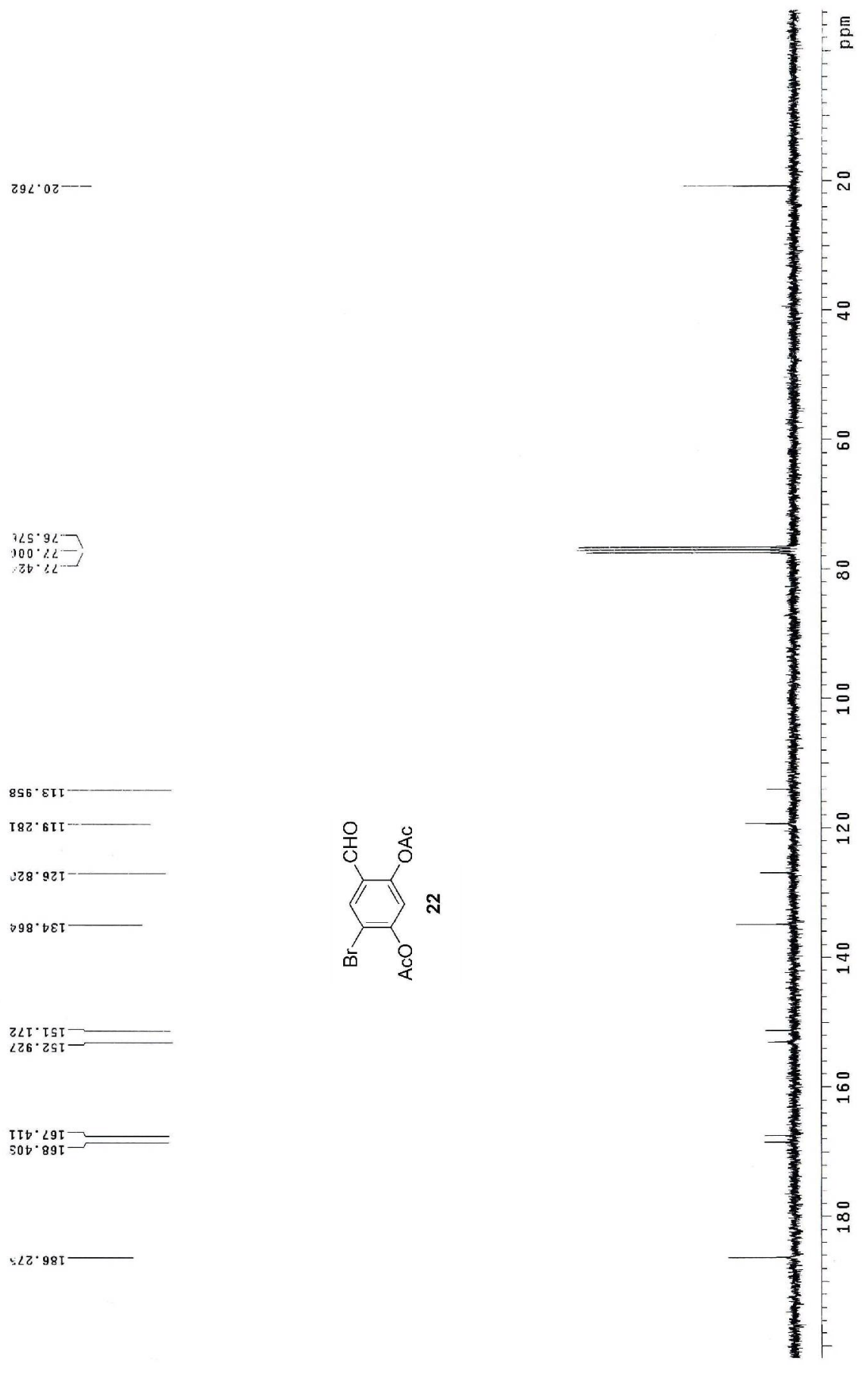

$Z \angle I^{\circ} T S T-$
$\angle Z 6^{\circ}-Z S T$

IIt: $29 \tau-$
$50 t \cdot 89 \tau-$

$325 \cdot 92=$
$100 \cdot 22=$
$30 \cdot 22$

$\because 22 \cdot 98 \mathrm{~T}$ 
$\operatorname{ct\tau } z-$

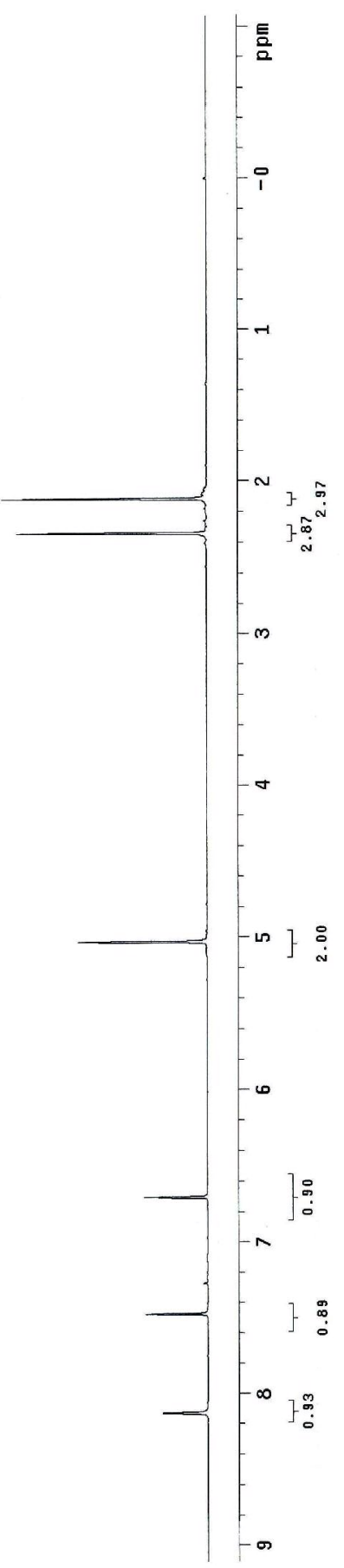


$502.02-$

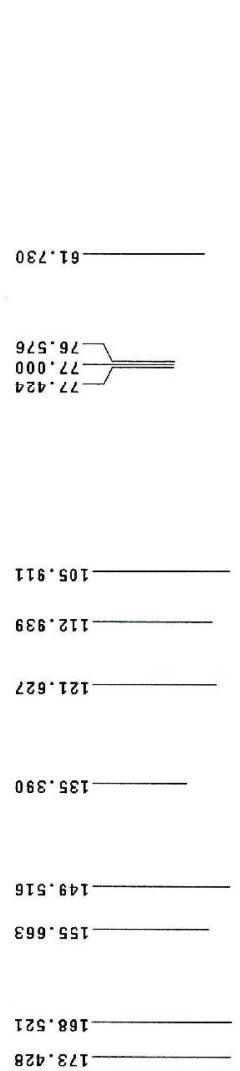

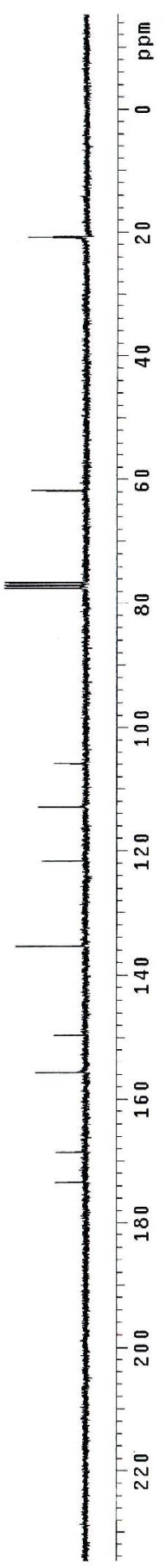




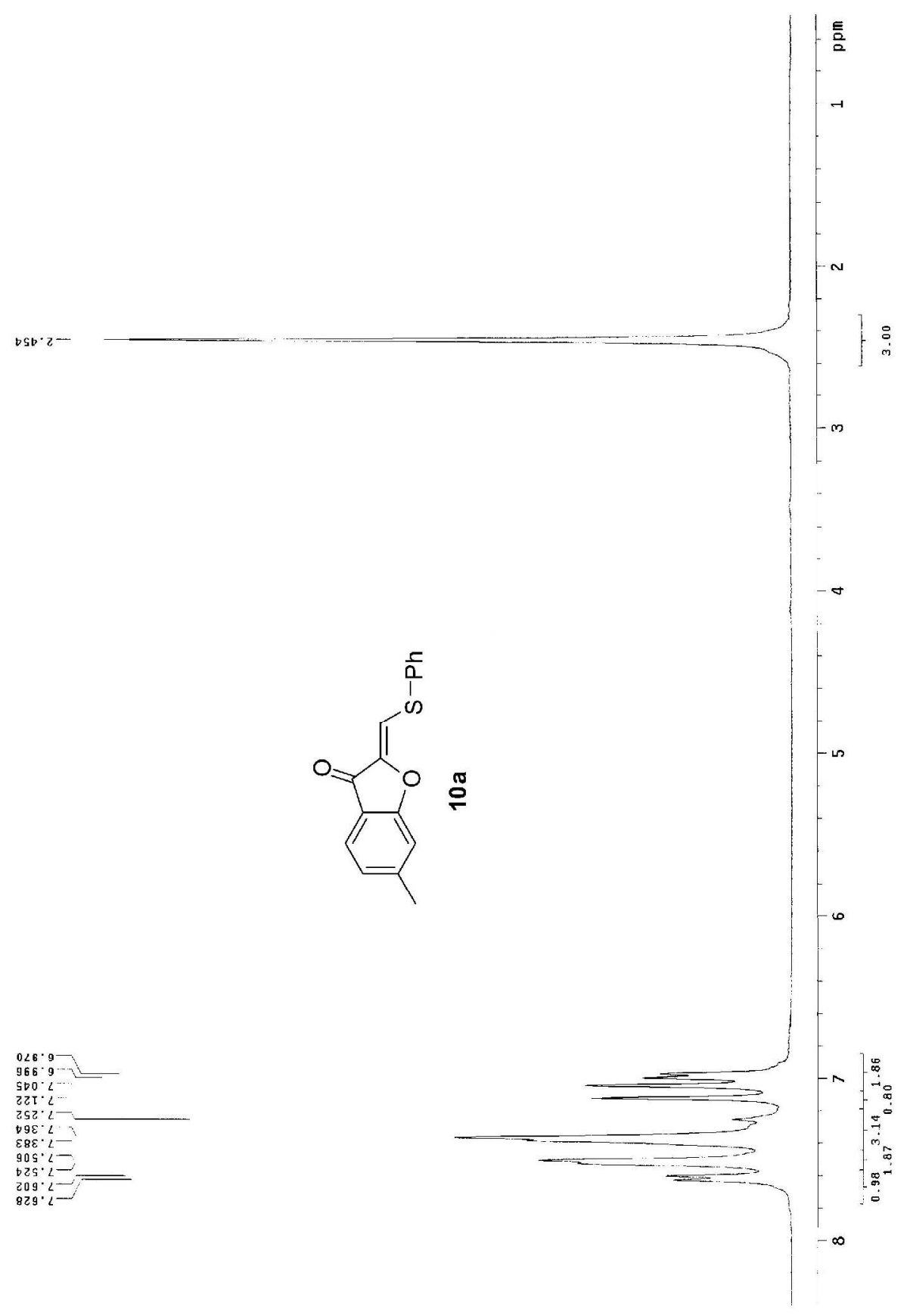




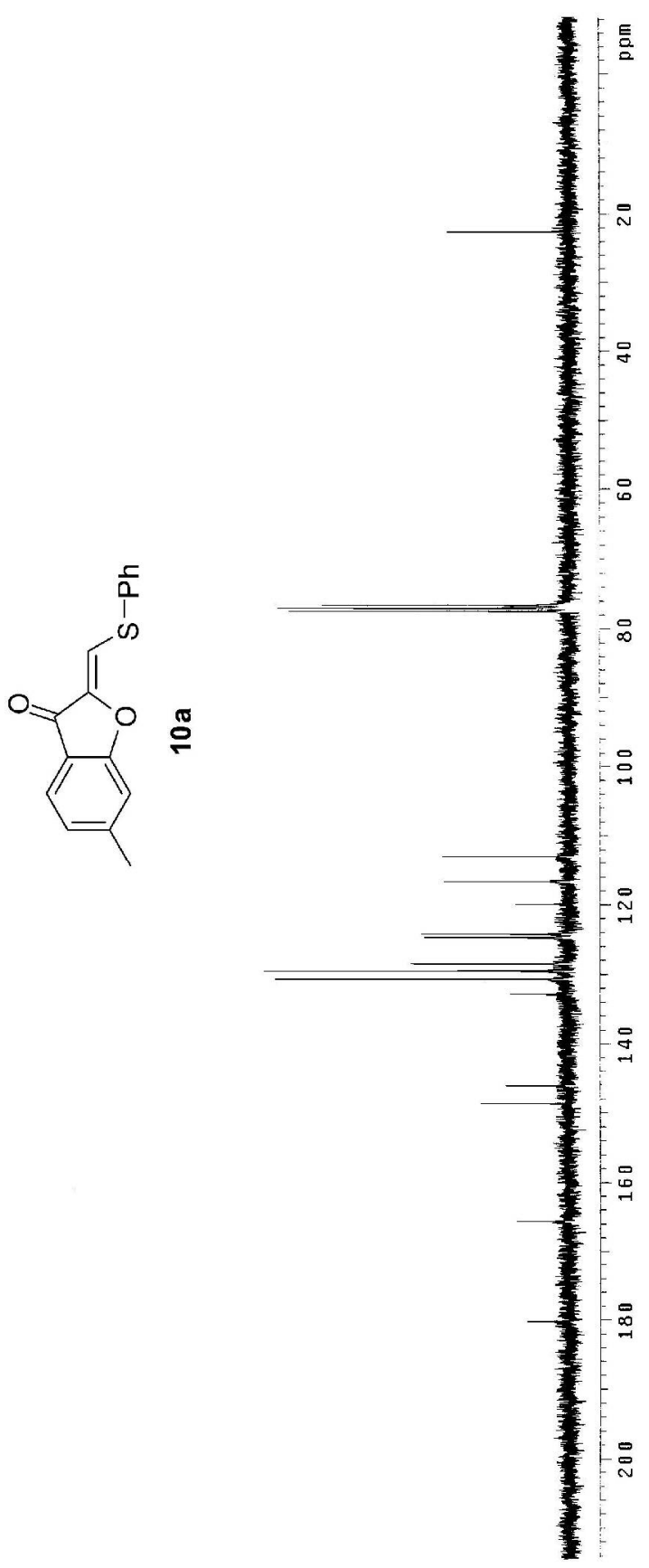

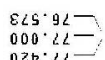

$886 \cdot 211-$
$829 \cdot 941-$

Tธ8.6 617

$69 \mathrm{~T} \cdot \mathrm{B} Z \mathrm{TT}$

$889^{\circ} \mathrm{DZT}$

E05. 62 I

E99.0ह

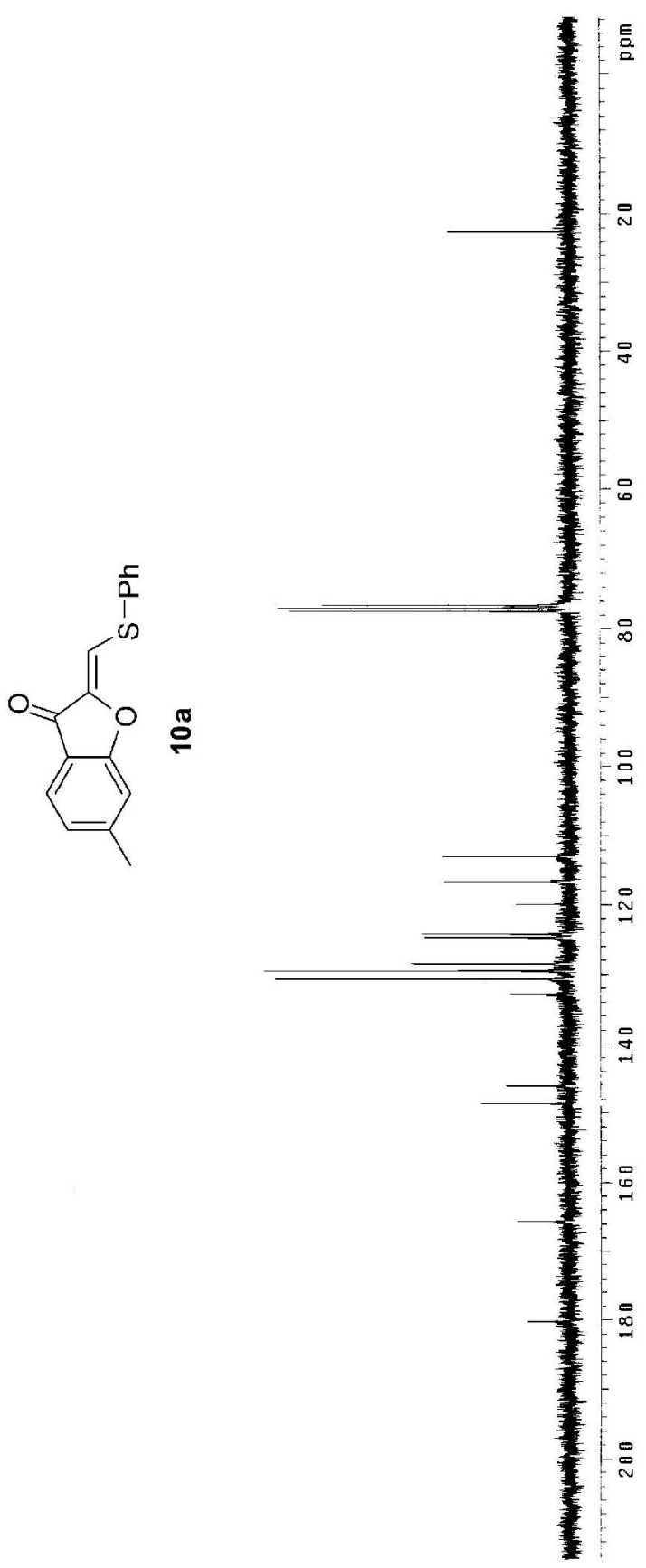

$06.50 I$
$059.80 I-$

$085.59 \mathrm{~T}$

SSI 081

\section{政}



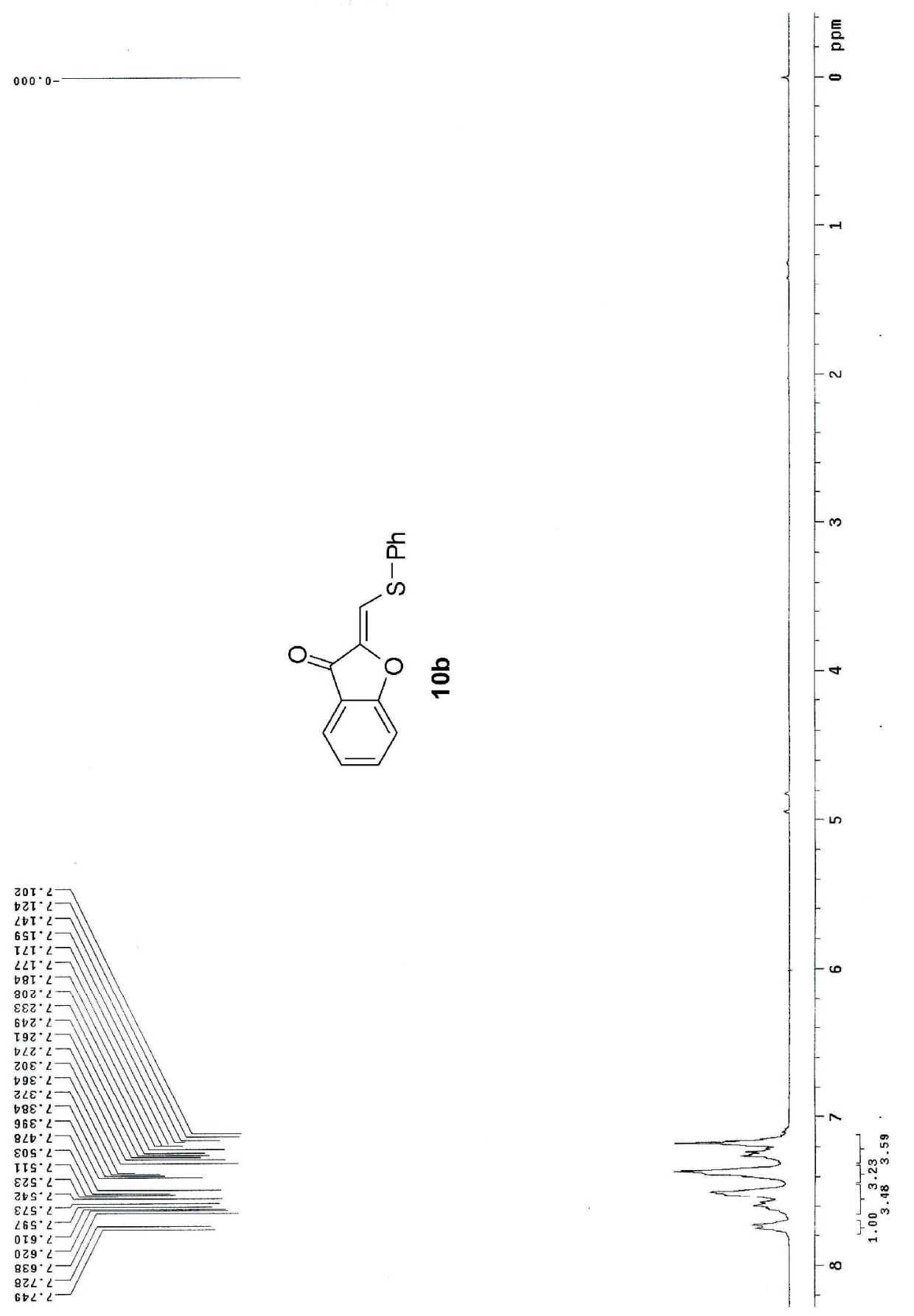


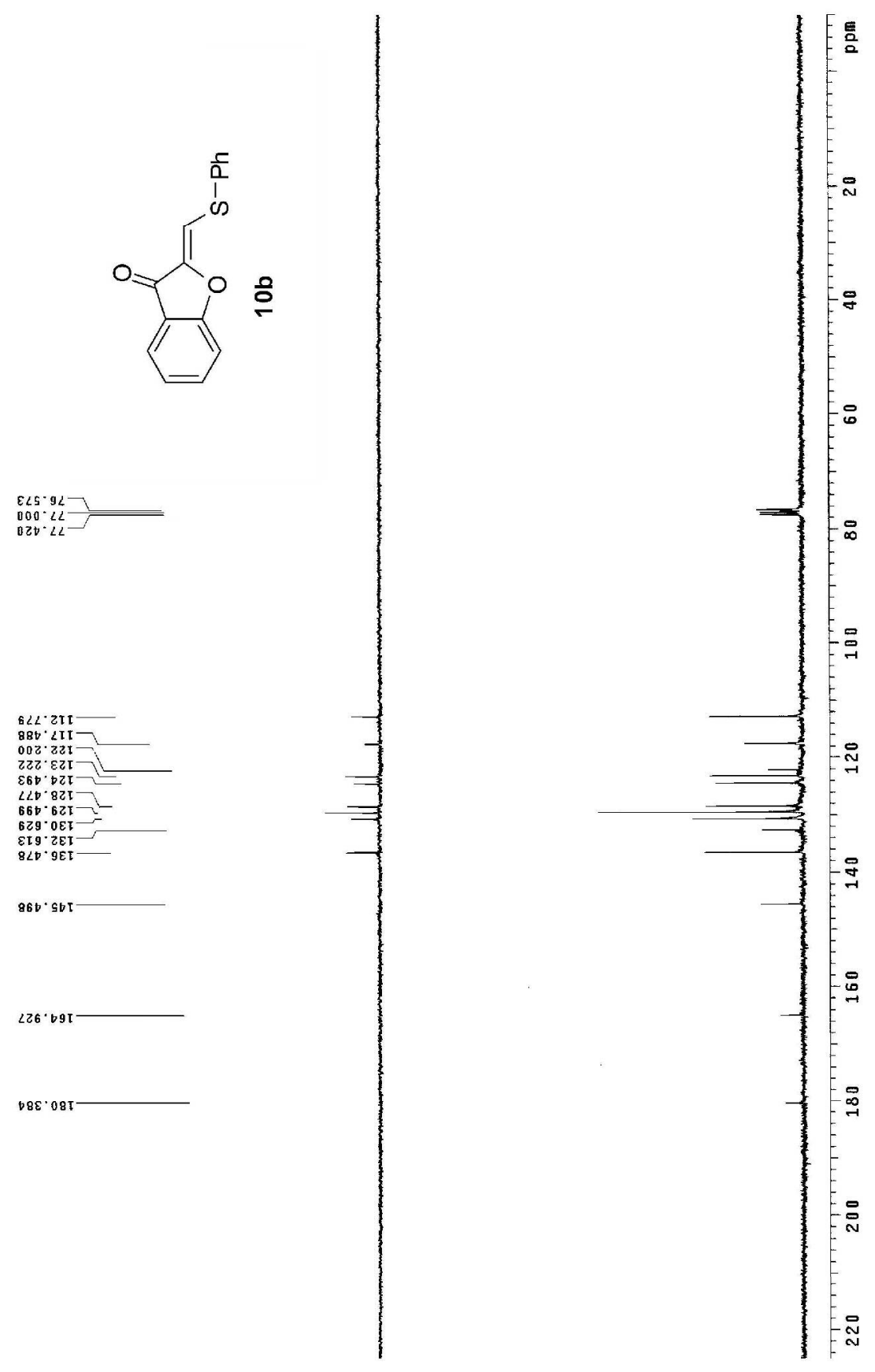


$800.0-$

$506^{\circ}$

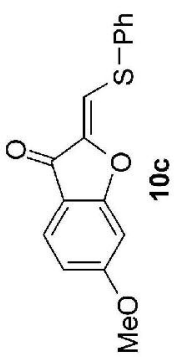

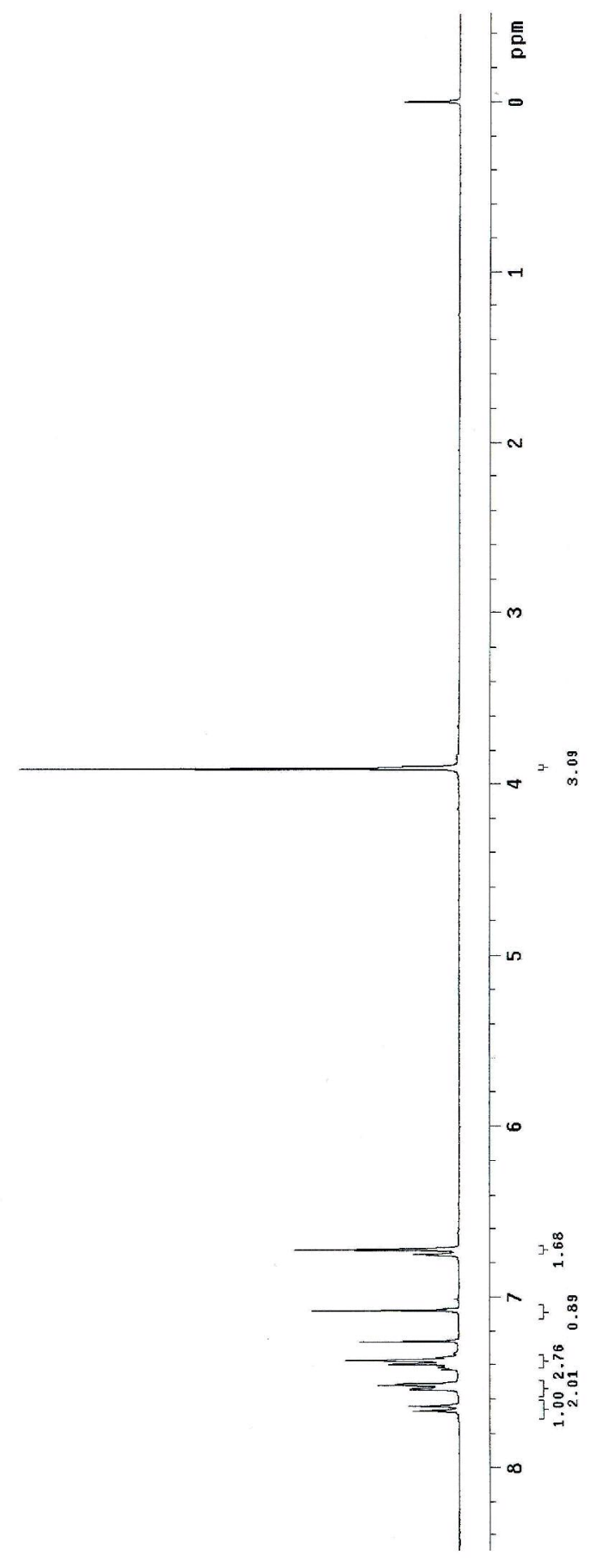



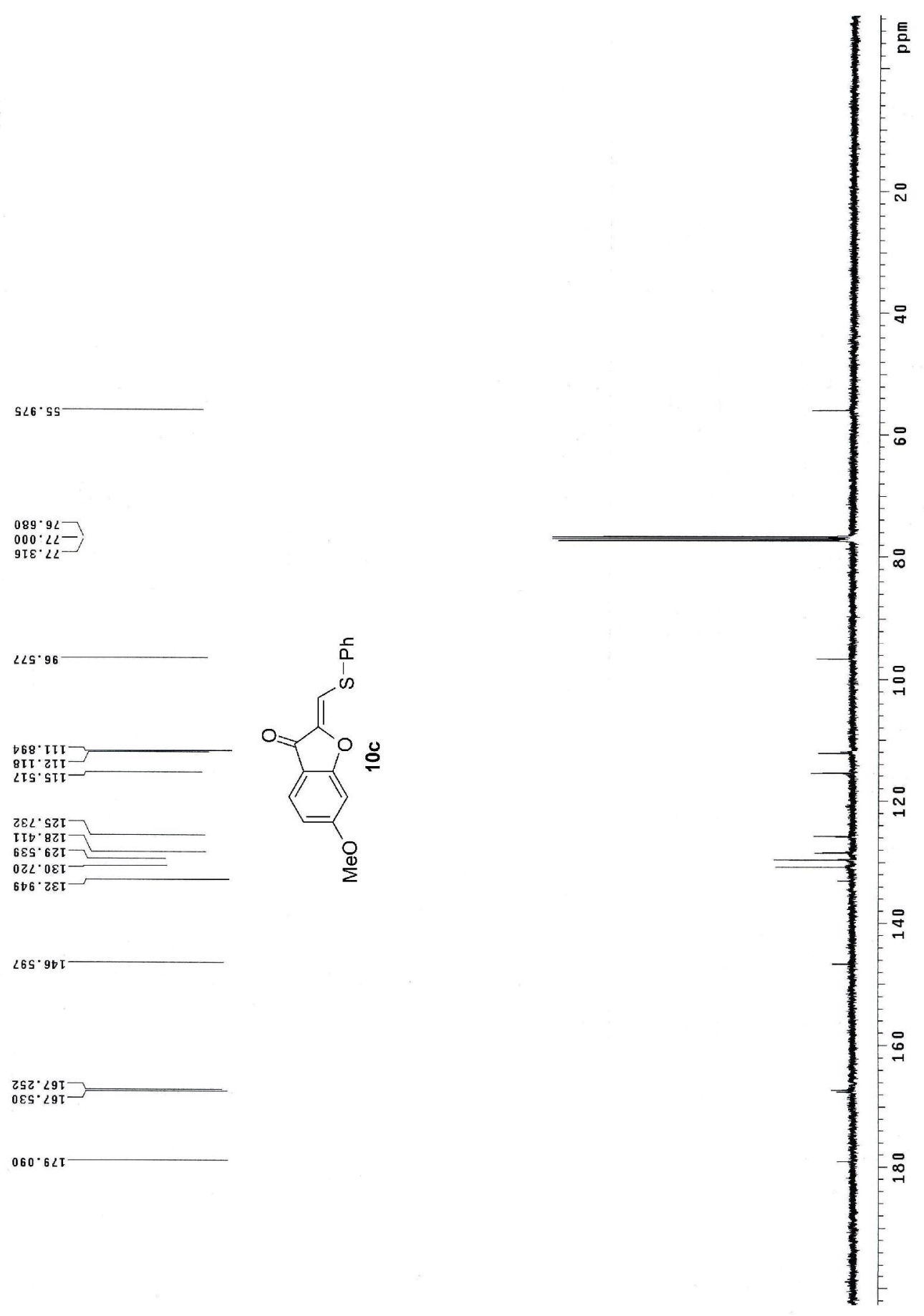
$210^{\circ} 0-$

$s<b^{\circ} z-$
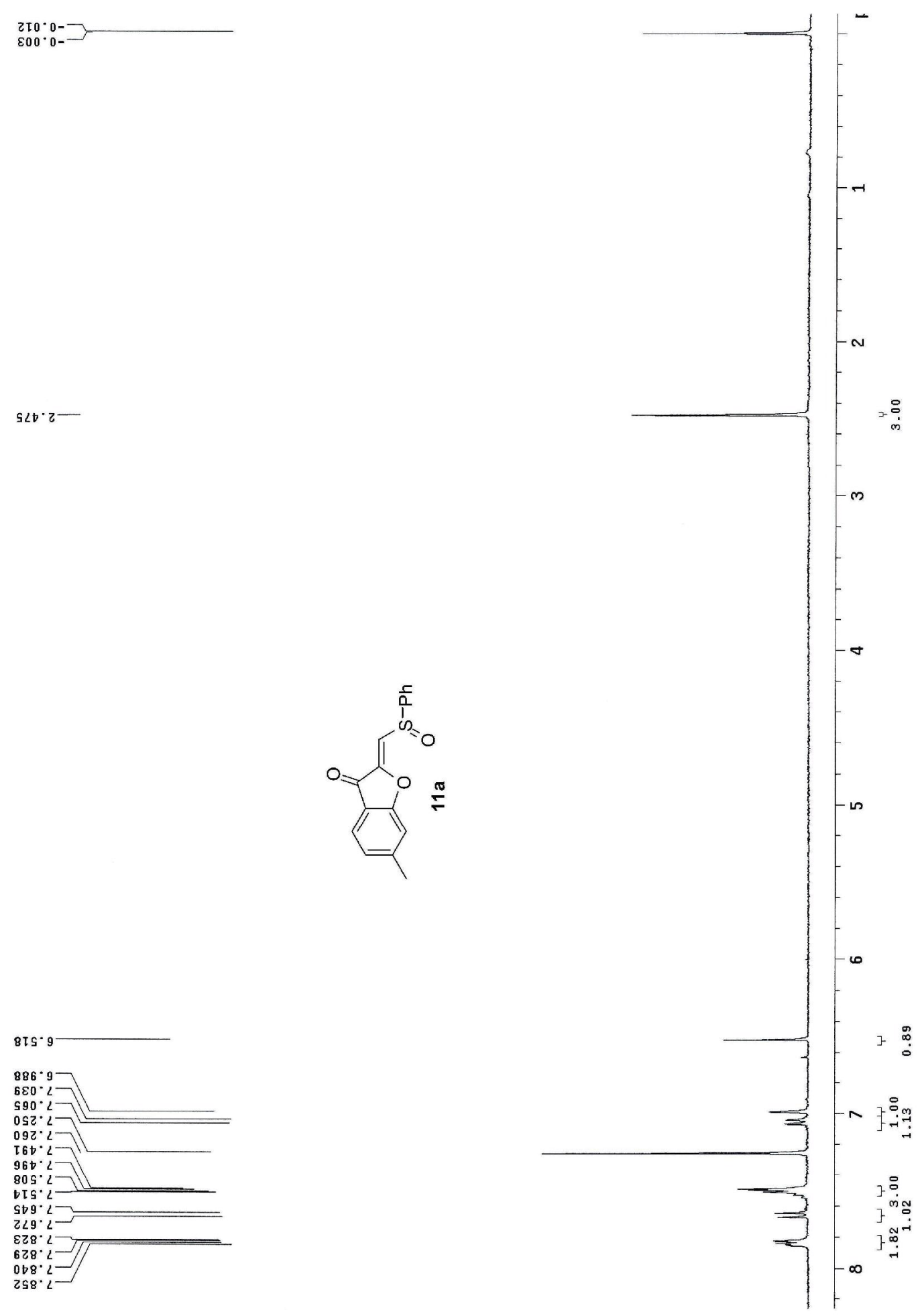
$208^{\circ} 22$

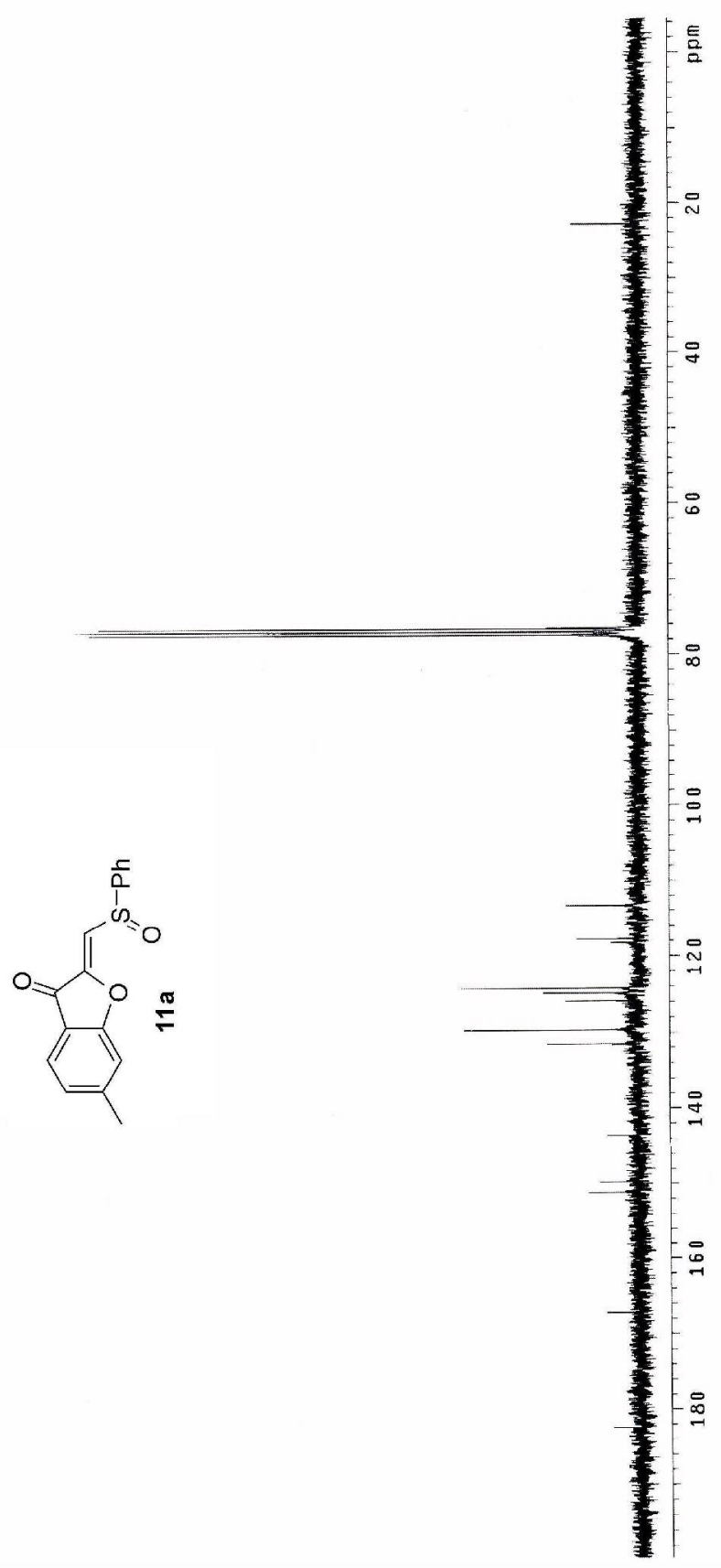

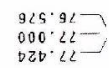

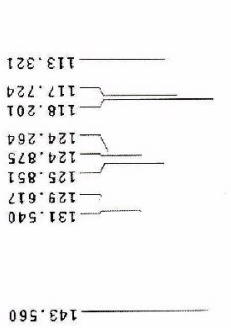

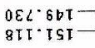

$\angle 6 T \angle 29 T-$

$\forall T H \cdot 28 T$ 


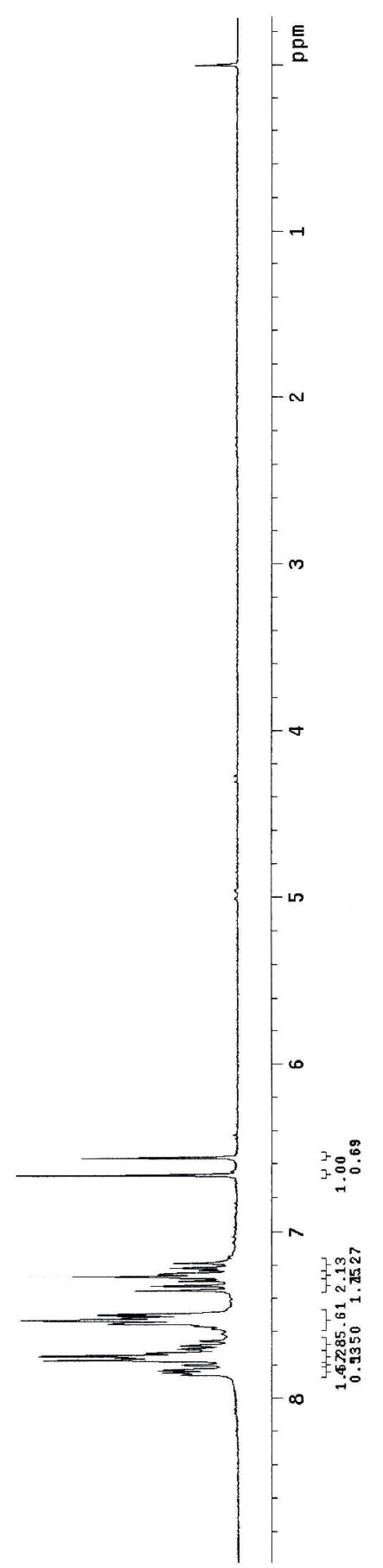


$260^{\circ} \varepsilon \mathrm{IT}$

(1)

$060^{\circ} 8 \tau \tau$

$6 z \mathrm{~b} \cdot 02 \mathrm{z}$

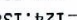

$B \varepsilon T^{\circ} b 2 \tau-$

$\angle 60 \cdot D Z T$

$\angle 0 T \cdot \mathrm{s} T-$
$\mathrm{ZLT} \cdot \mathrm{SZI}-$

$699 \cdot 535$

I I $62 \mathrm{I}$

T9.6 62

ว85. โย

${ }^{68 \varepsilon} \cdot 8 \varepsilon \mathrm{I} \square$

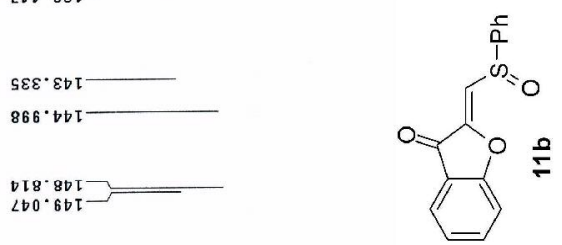

$\underset{108 \cdot 995-}{88.997-}$

$536 \cdot 381$

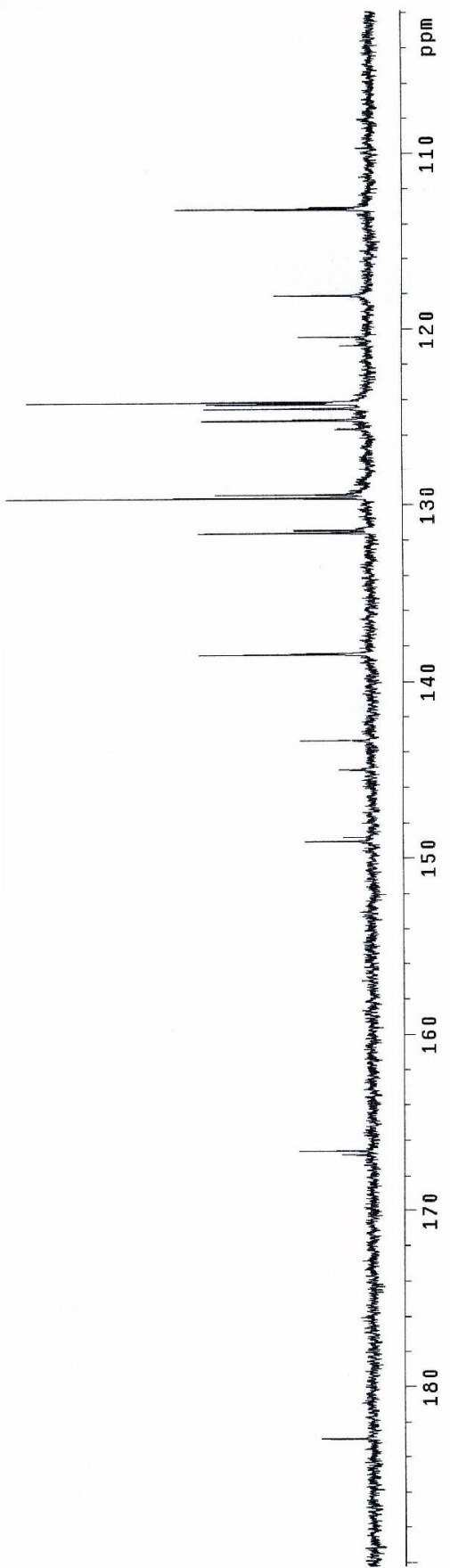




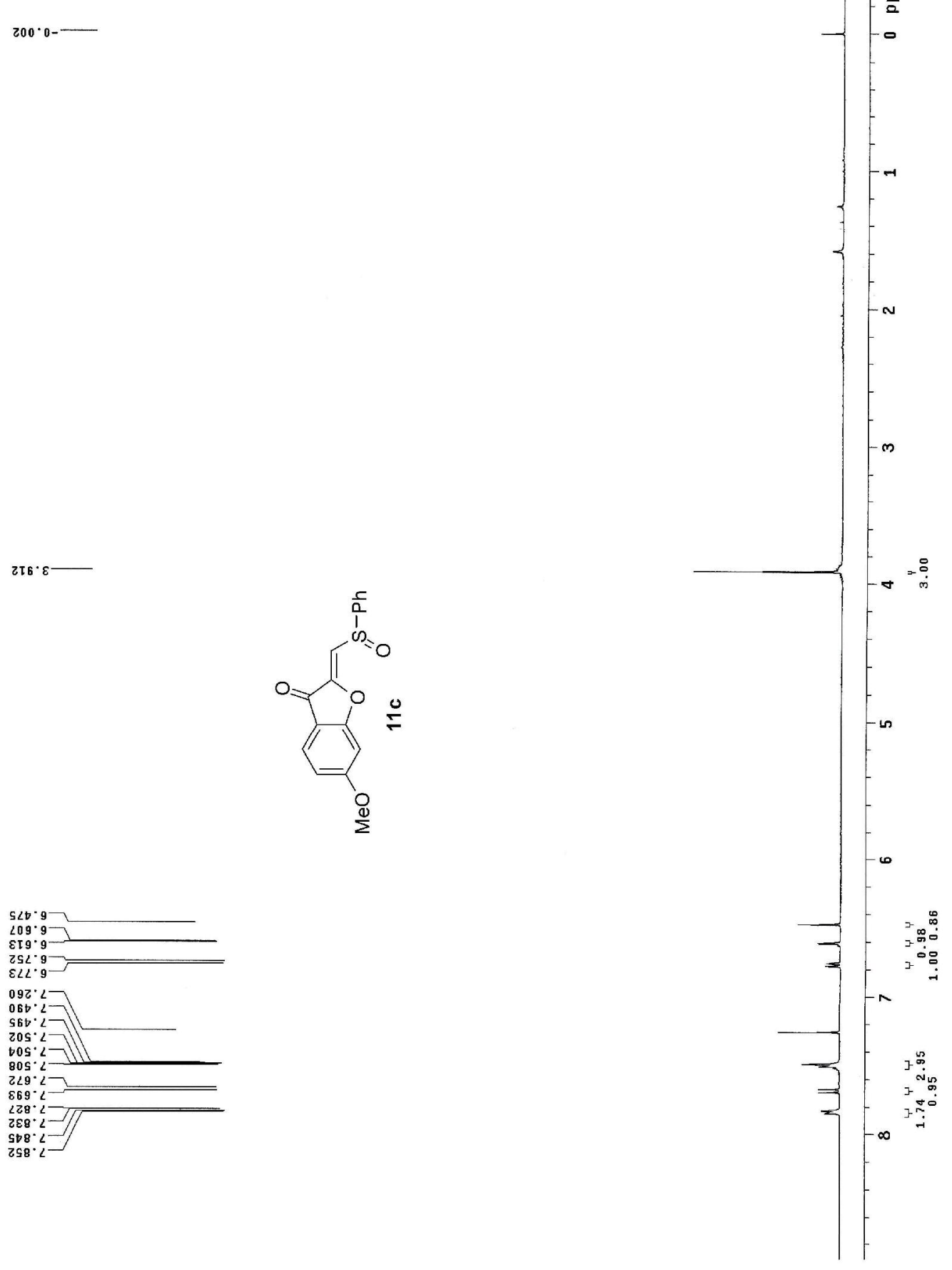



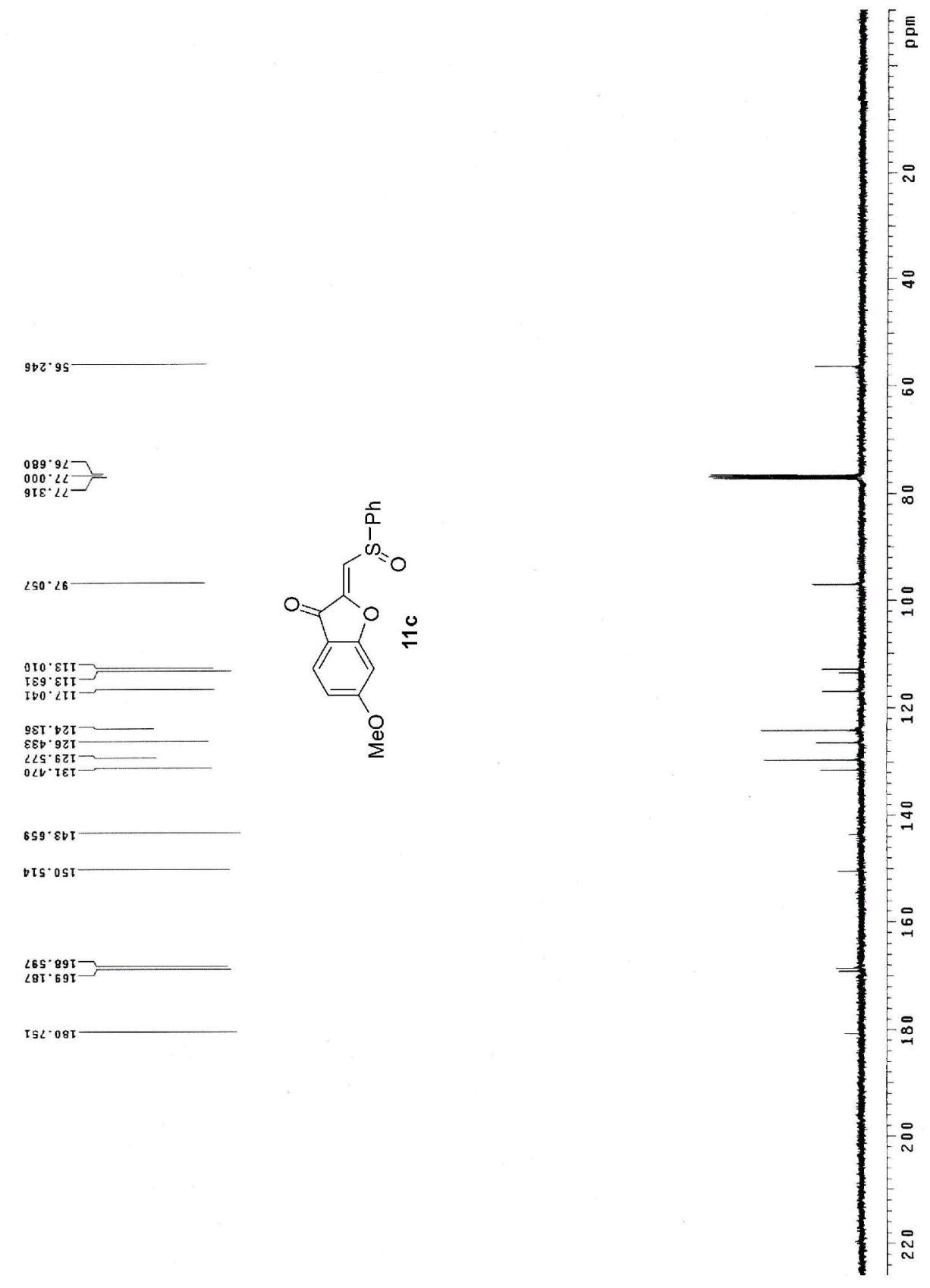
$000 \cdot 0$

$088^{\circ} z-$

$066 \cdot 2$

$299^{\circ}$
$969^{\circ}$

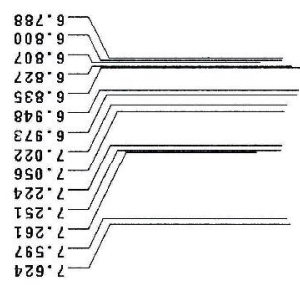

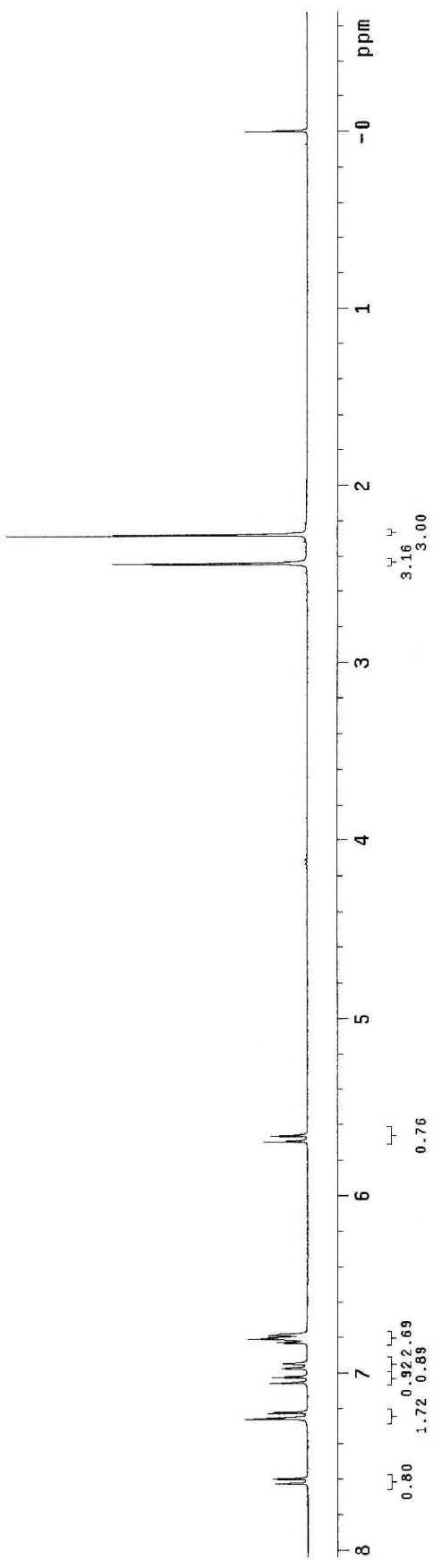


${ }_{970}^{650} \cdot 22=$

089.92 二

05.10T -

$028 \cdot 0 \mathrm{TI}-$

$89 \varepsilon^{\circ} \varepsilon 1$ -

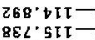

$680.91 \mathrm{~T}$

$\angle E t) b z t$

$=$

$88 \mathrm{I} \cdot 62 \mathrm{I}$

GsE. $\mathbf{s s t}$

GE8. ISt

$5 \angle 8 \cdot 89 \mathrm{I}=$
$6 \div \angle \cdot 0 \angle \mathrm{I}=$
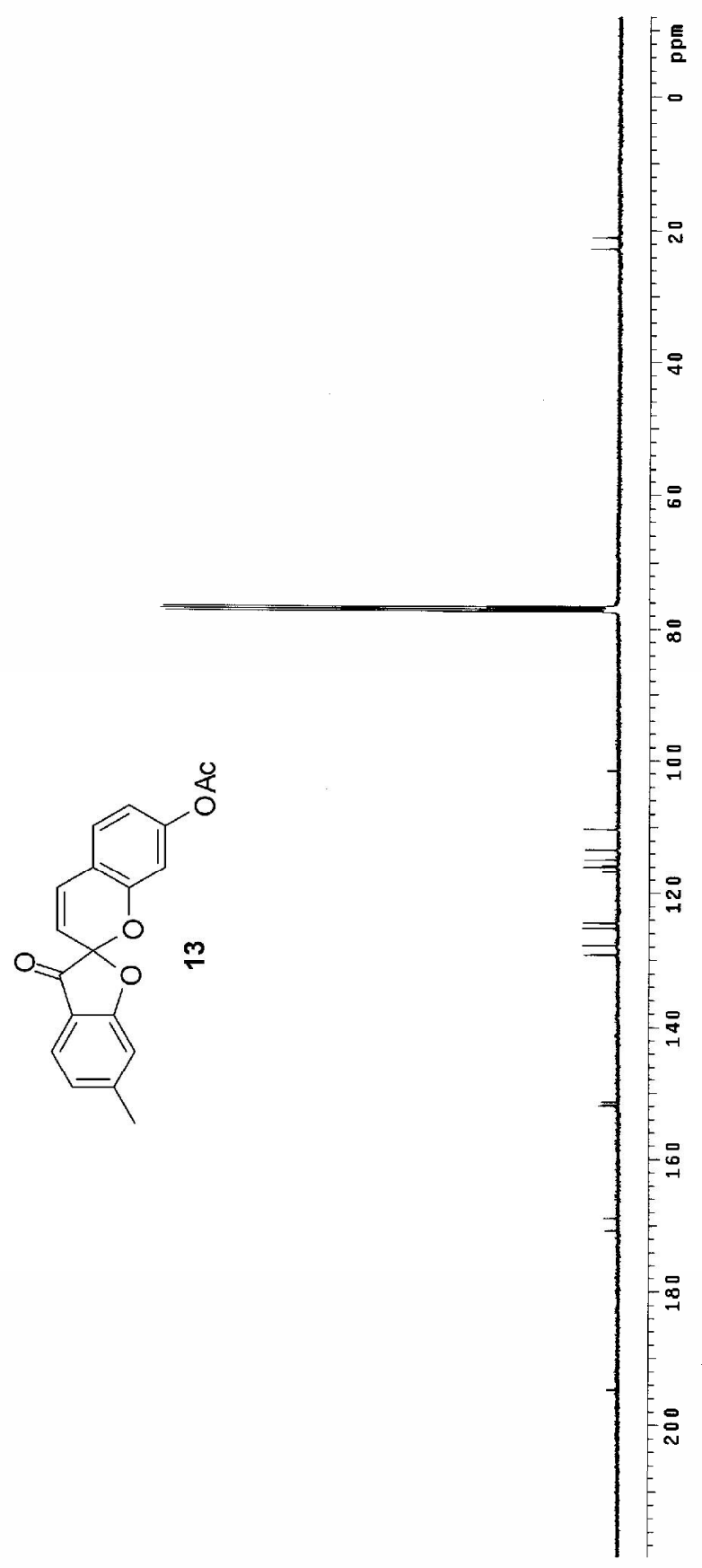
$000^{\circ}-0$
$800^{\circ}$

$582 \cdot 2-$

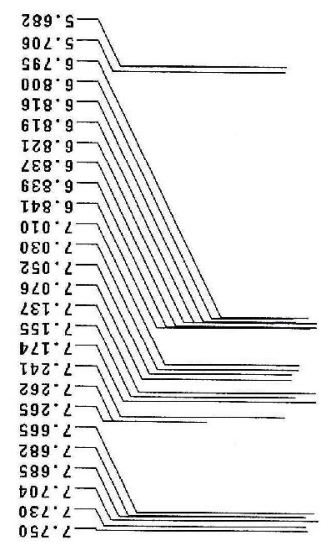

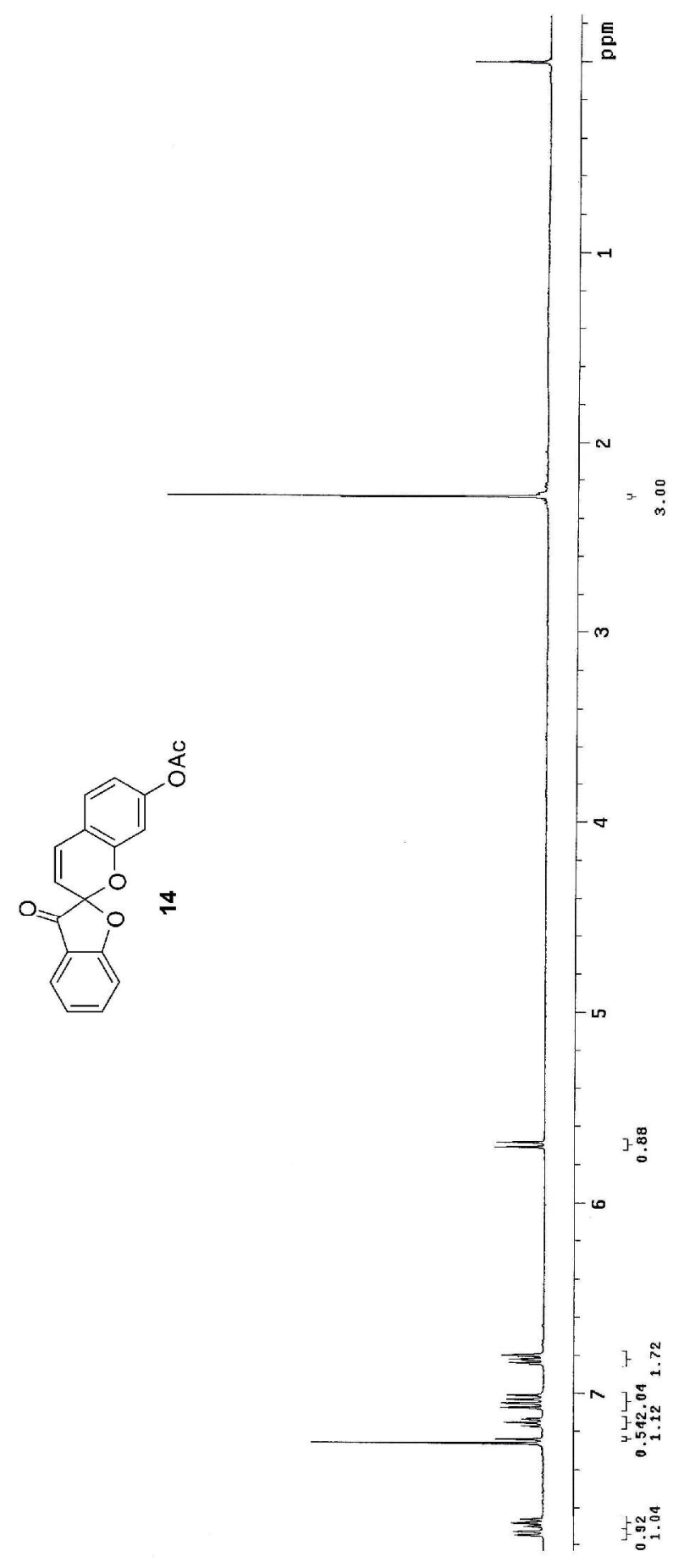


$0<0^{\circ} \tau z$

$089 \cdot 9 \ell=$
$000 \cdot \angle L-$

$\underset{918<L-}{000}<2$

เ9T โ โ0I

วTE.0II-

$6 \triangleright \varepsilon^{\circ} \varepsilon \mathrm{II}$

$\angle \varepsilon 9^{\circ} \bullet I I$

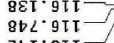

Zt. 811

हD6. 2 . I

$9 \angle 5.5 Z I-$

VG8. $\angle Z I-$

I $6 b^{\circ} 68 \mathrm{I}$

$6 \angle Z^{\circ}$ ISI
$688^{\circ}$ ISI

$288^{\circ} 89 \tau-$
$262^{\circ} 0<\tau$

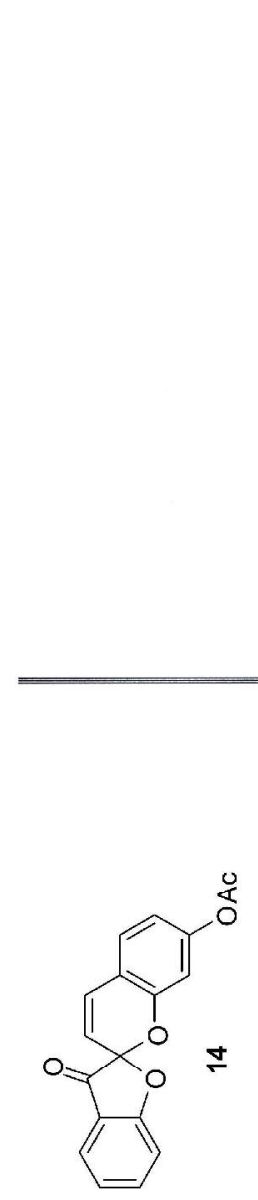

I0b. 56 T

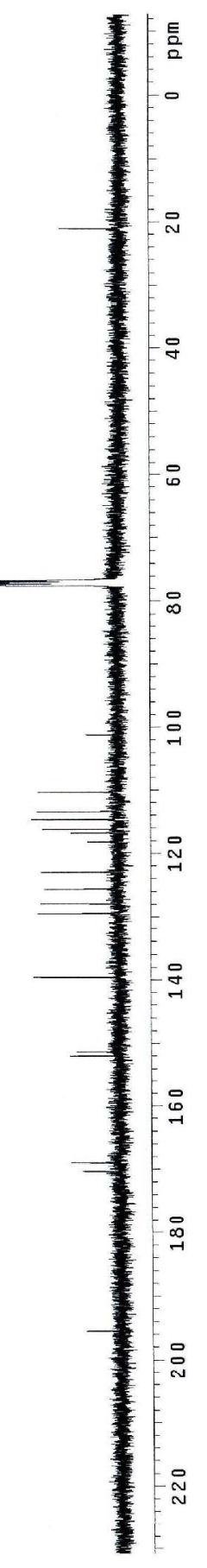


工 $00.0-$

$200 \cdot 0$
500.0

$\varepsilon 82 \cdot z$

$โ 88^{\circ} \varepsilon$

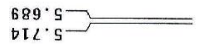

$\angle 2 t \cdot 9$

280
$2 \angle 9 \cdot \theta$

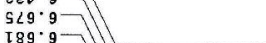

$\left.\begin{array}{l}{\left[89^{\circ} \cdot 9\right.} \\ 269.9\end{array}\right]$

269.9
$80<.9$

008.9

L 208.97

$878^{\circ} 9$

SSO $0^{\circ} \mathrm{L}$

$0 \varepsilon 2 \cdot 2-$

os : 2 - 20

$\begin{array}{lll}892 & 2= \\ 992 & 2=\end{array}$

$992 \cdot 2$

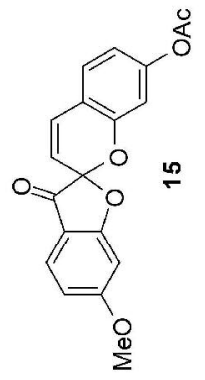

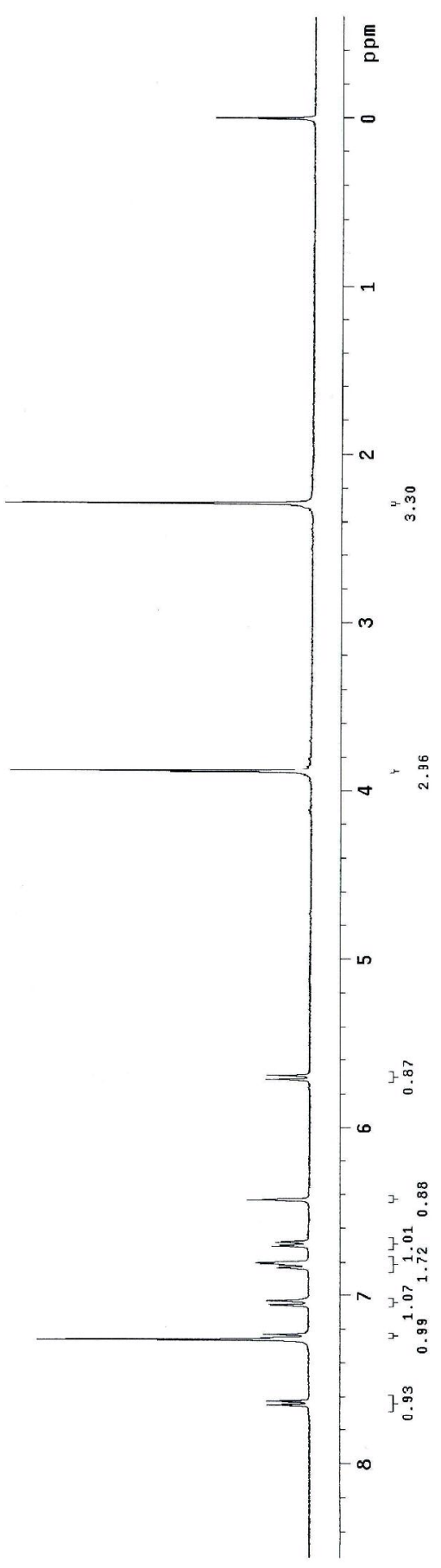


$890 \cdot 12$

470.95

$689.92=$
$000.22=$

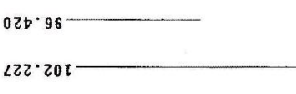

9TE. OT

T08. 2 It

<28. $551-$

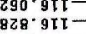

:zв:

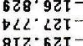

—

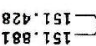

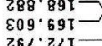

$668^{\circ} 265$

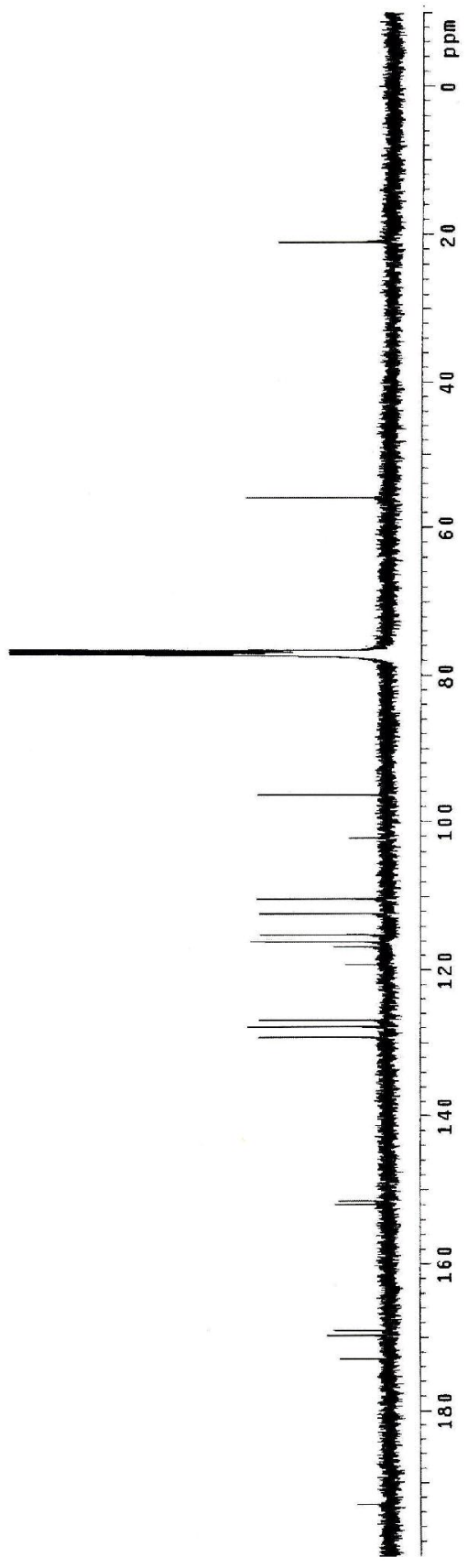


$000 \cdot 0-$

$\tau \forall \varepsilon \cdot 2-$
$9 \forall t \cdot 2-$

$602 \cdot \mathrm{s}$

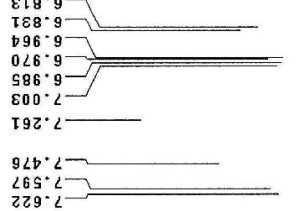

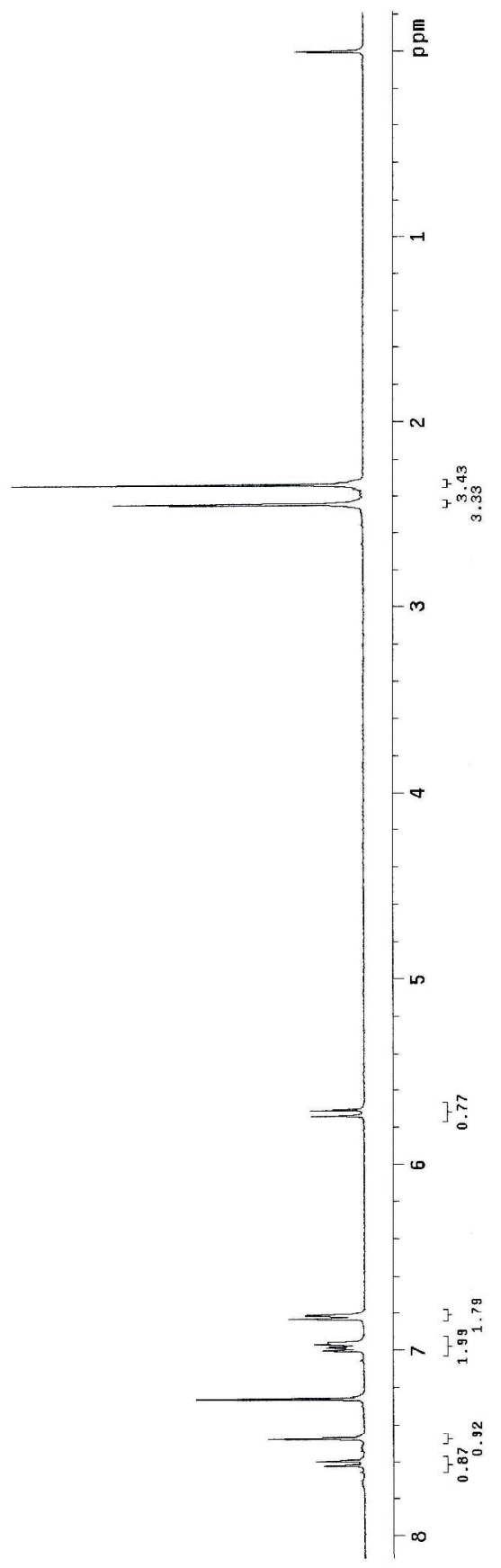


602.5
$802 \cdot 5$

$\underset{\tau \in 8^{\circ}}{\varepsilon I 8^{\circ}}$
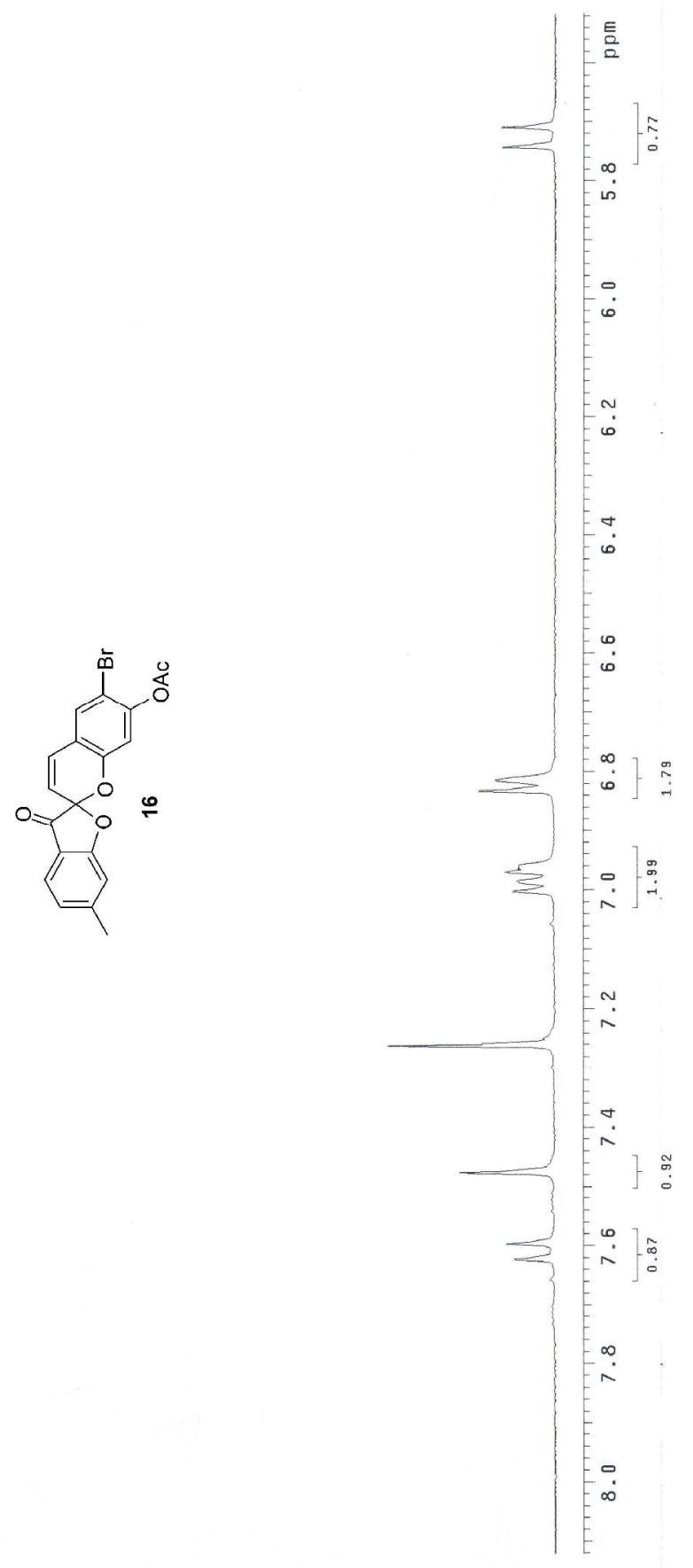

$265^{\circ}-2$

$496.9-$

$026^{-9}$
$586^{-9}$
$800^{\circ}-$

โ98.2

$9 \angle t \cdot 2$

229.2 


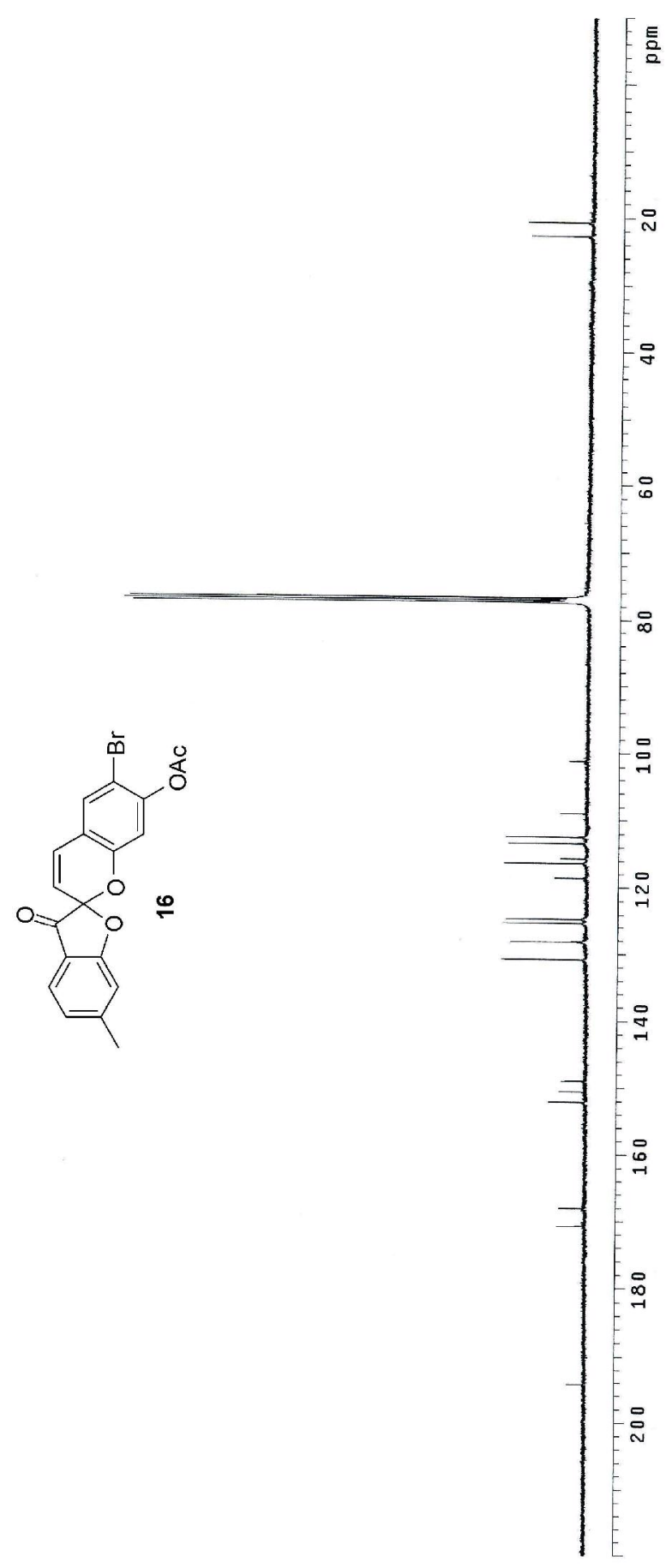

$089 \cdot 92$
$000.2 L$

Eัเ โนT

$\$ 66.801-$

DSD'ZIT

SOg.st

908.9 I I

595.8I I

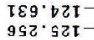

061.821

STR 0 ET

$600.6 \mathrm{tT}$

TIS. OST
0 TI. $25 T$

$520 \cdot 892$

$50<\cdot 0<1$

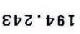

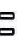


$100 \cdot 0$

$25 \varepsilon^{\circ} 2$

$0 z 2 \cdot 5$
052

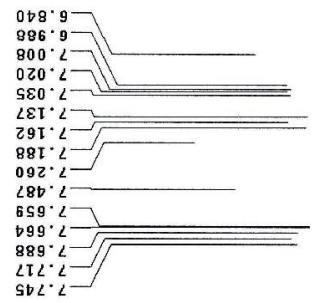

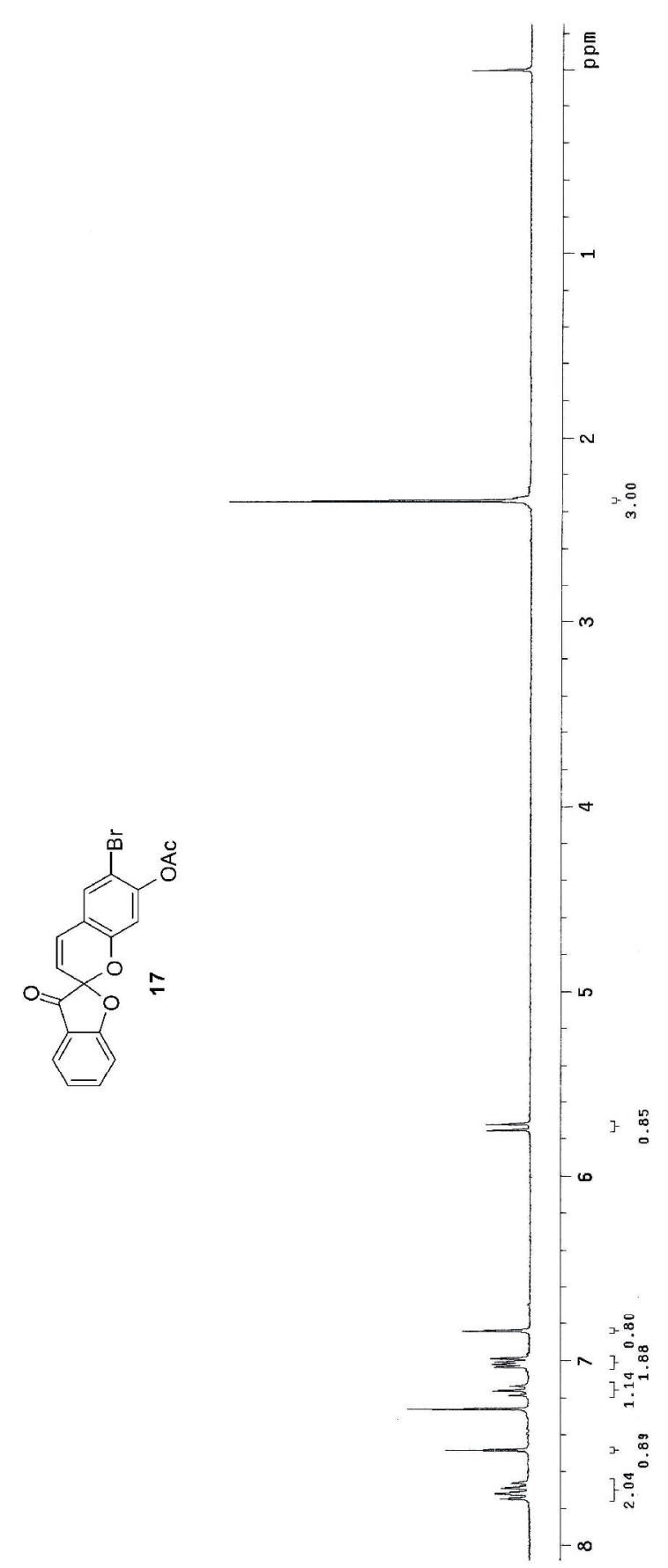


$0 z 2$

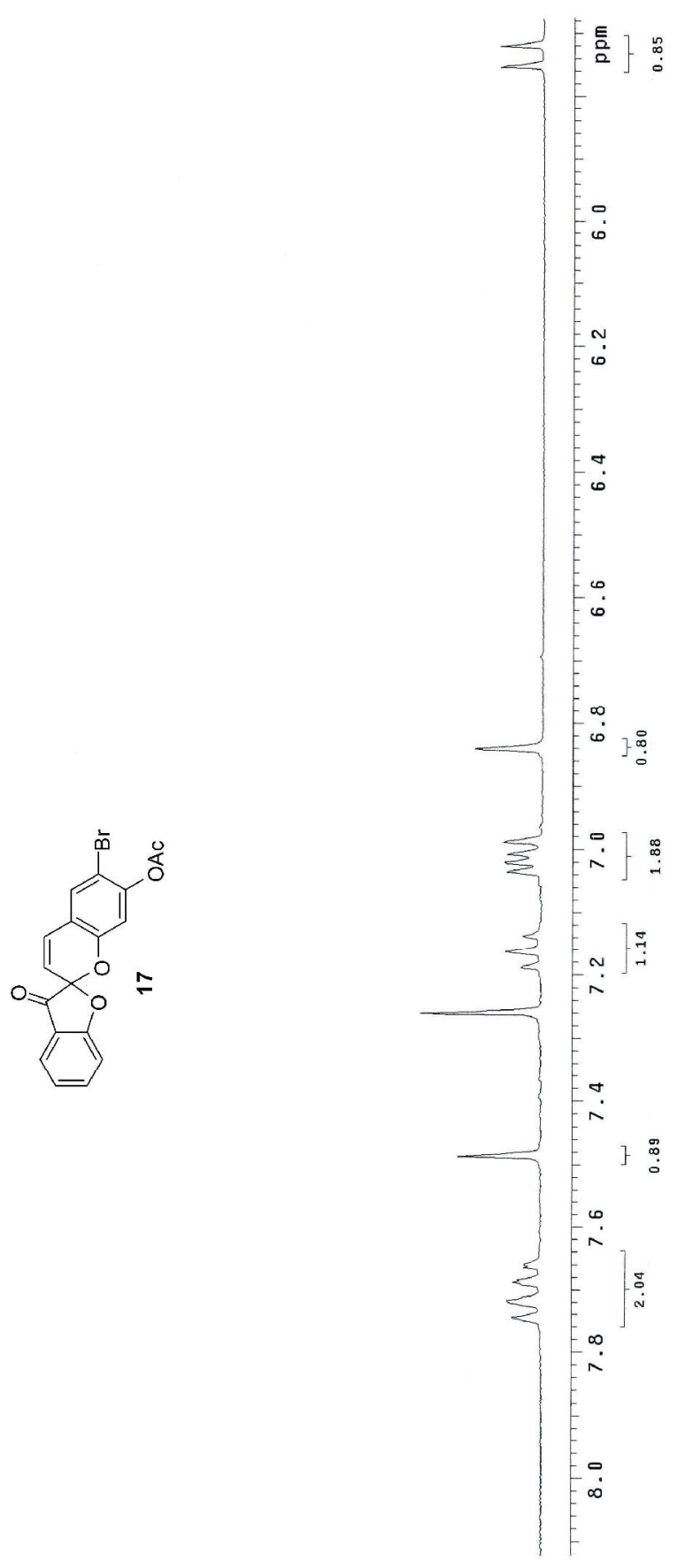




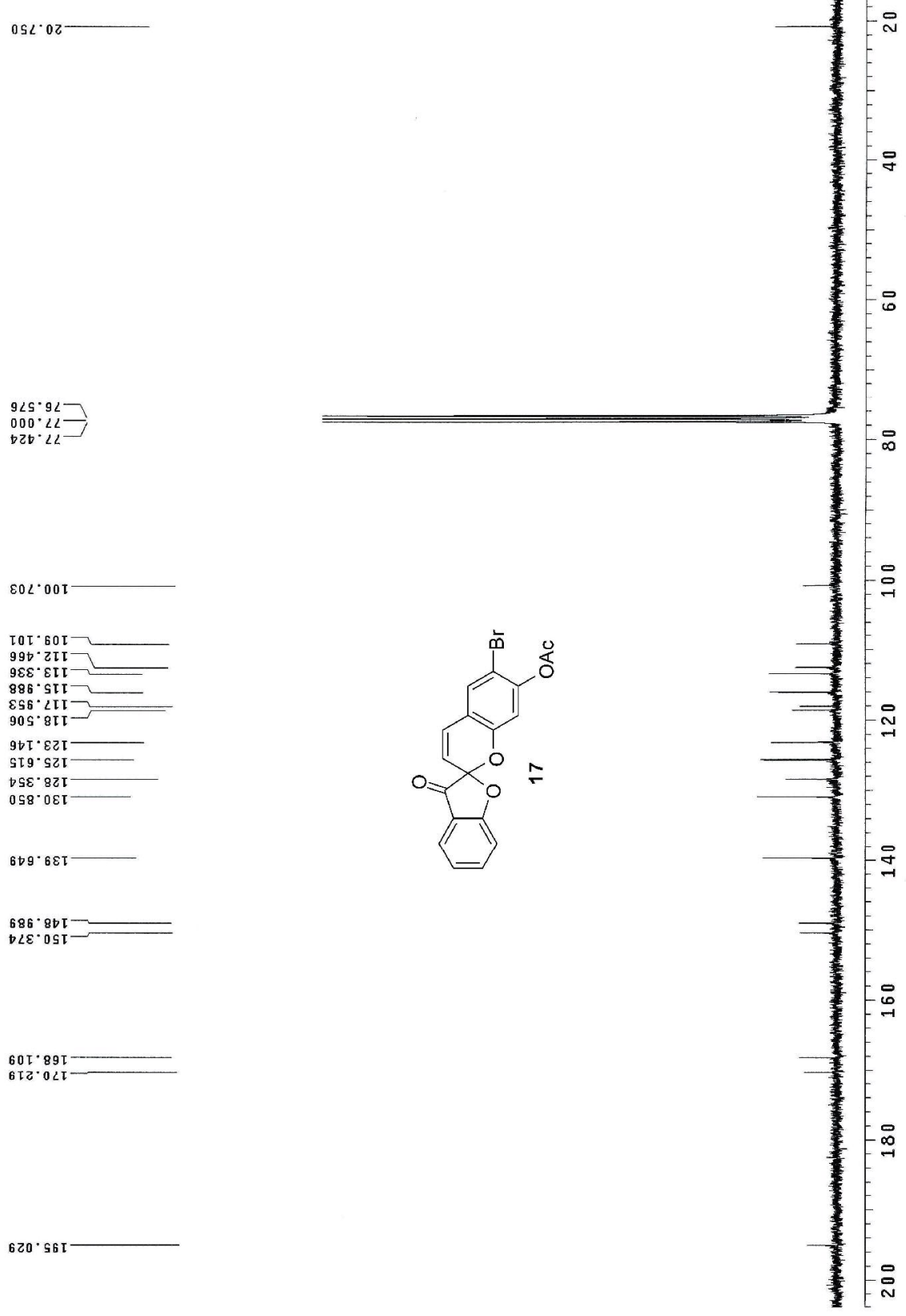


$200 \cdot 0-7$

$z+\varepsilon \cdot z-$

$988 \cdot \varepsilon-$

${ }^{0 E L} \cdot \mathrm{s}$

$8 Z t \cdot g$
$58 b \cdot g$

$586 \cdot 9$

$089 \cdot 97$
$889 \cdot 9$

$602 \cdot 9$
$\angle \mathrm{L} L \cdot 9$

$908 \cdot 9-$

200.2

$052 \cdot 2$ D

$6 \angle b \cdot 2$

$029 \cdot 2$
$609 \cdot 2$

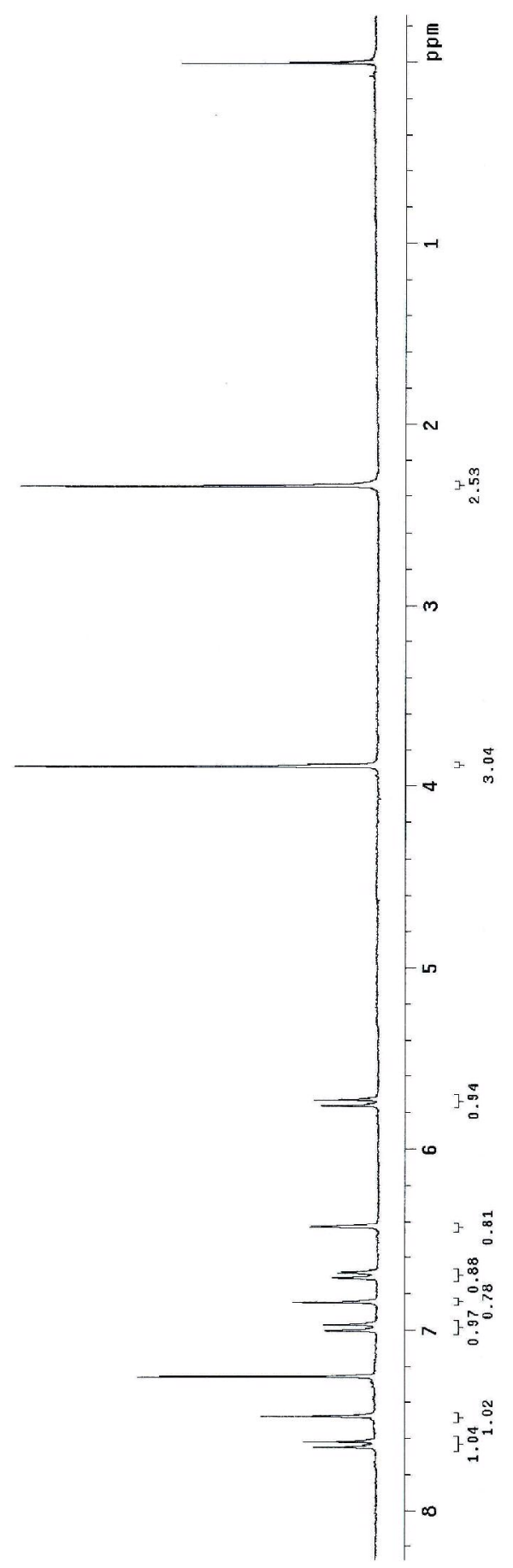


$85 L^{\circ} 02-$

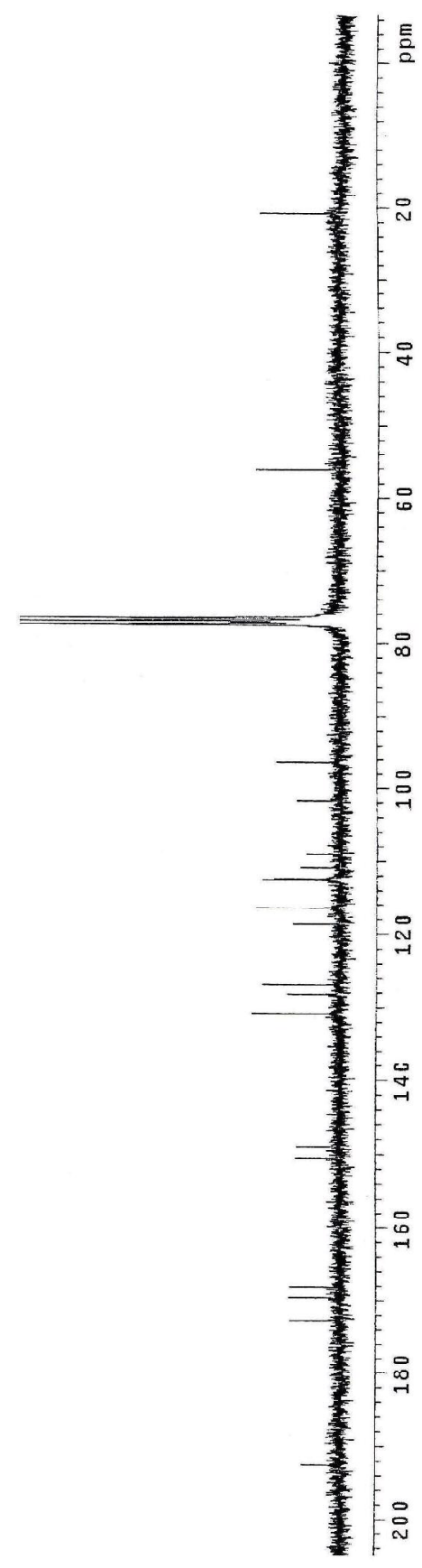


${ }_{t} 00^{\circ} 0-\longrightarrow$

${ }_{62 \varepsilon} 18: 2=$

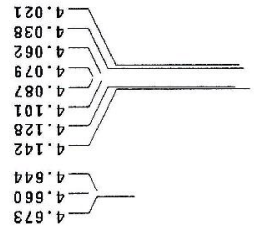

550.27

$080.2=$
901.2

T9. 2

${ }_{285} 25$

285.2

97920

$029 \cdot 2=$

$879 \cdot 2=$

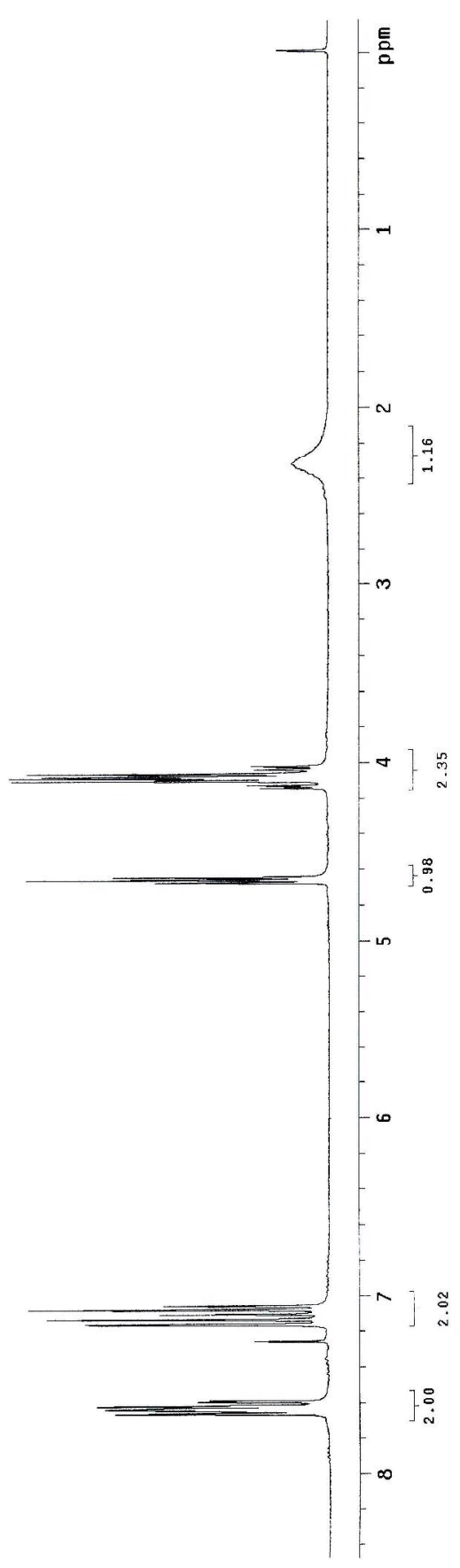


$S \varepsilon 9^{\circ} \div 9$

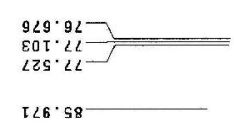

$0<t \cdot \varepsilon \tau \tau$

$261 \cdot 12 I$

${ }_{80 t}^{210} \cdot 02 t$

$\varepsilon \varepsilon \varepsilon^{\circ} \cdot 8 \varepsilon \downarrow-$

$\llcorner 0 z \cdot \varepsilon\llcorner\tau-$

$28 \varepsilon \cdot 002$

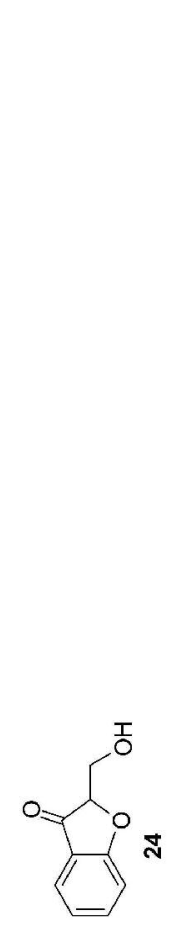

A

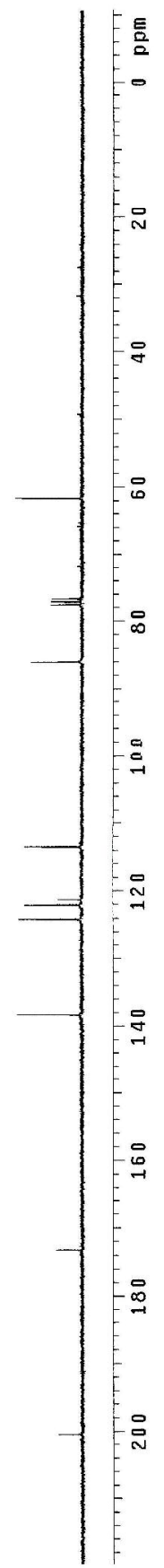




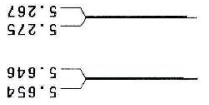

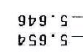

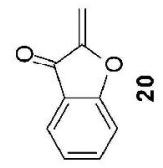

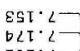

$202 \cdot 2$

$909.2=$

$\angle 59 \cdot 2$

$881.2=$

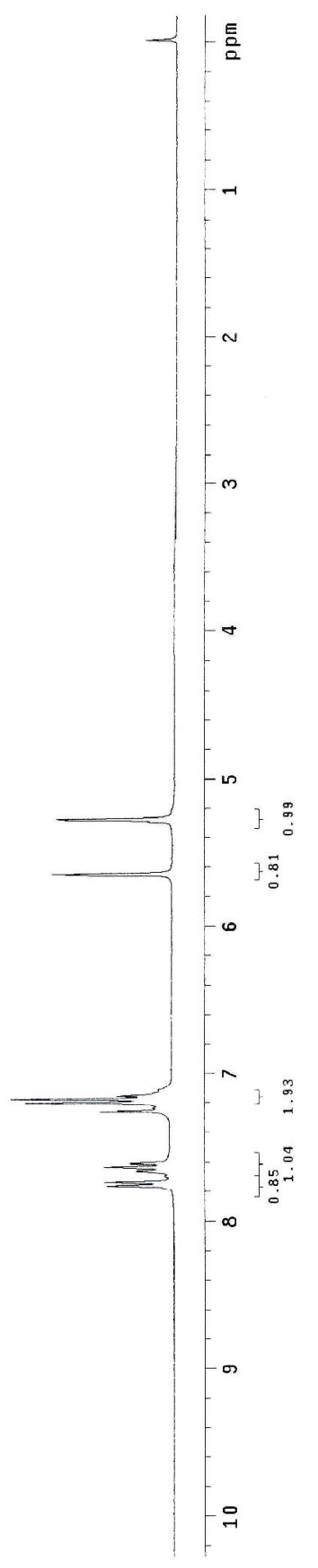


$925 \cdot 92-$
$000 \cdot 22=$
$b 2 t \cdot 22-$

$\varepsilon s 2 \cdot 26$

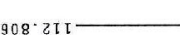

728.5217

$8 \circ 2 \cdot 8 z I=$
$8 \circ 8 \cdot 6 z I=$

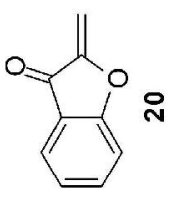

$\varepsilon \angle 5^{\circ} \angle \varepsilon I$

$390^{\circ} 60 T$

โระ. $99 \mathrm{~T}$

$956^{\circ} 281$

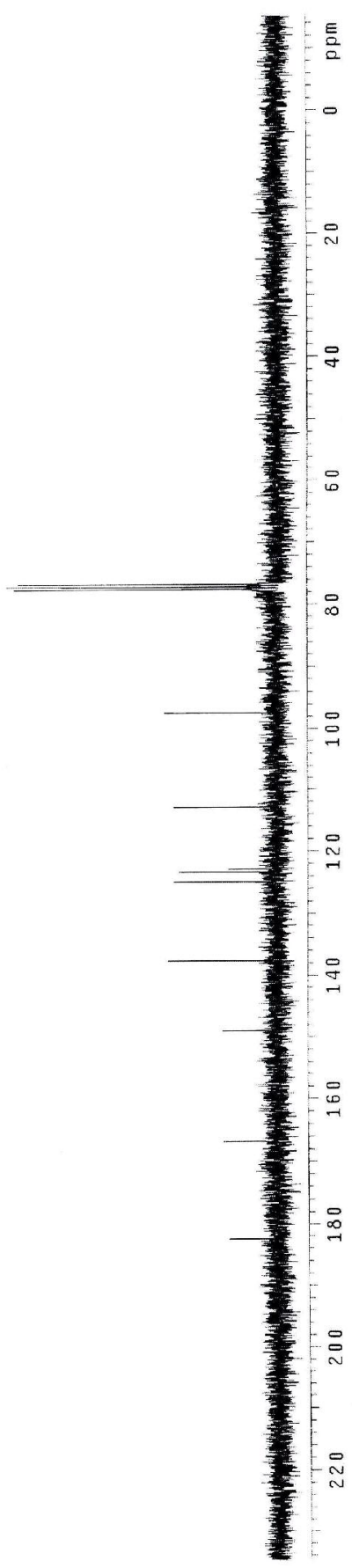


${ }^{1000^{\circ}} 0^{\circ-}$

$010^{\circ} z-$
$810 \cdot 2$

$620^{\circ} 2$

$280^{\circ} \mathrm{z}-$
$\varepsilon \Delta 0^{\circ} \mathrm{z}$

$\angle 50^{\circ} 2$

$920^{\circ} \cdot 2-$

เ282.

obz:z-11

$88 \cdot z-\overline{ }$

เा $\cdot 2$

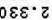

I $68^{\circ} \cdot z$

$9 \mathrm{~b} 6 \mathrm{G}^{\circ} \cdot$

$\mathrm{D} 56^{\circ} \mathrm{z}$
$\mathrm{S} 9 \mathrm{6}^{\circ} \mathrm{z}$

年

$2 \varepsilon \tau \cdot \varepsilon$

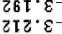

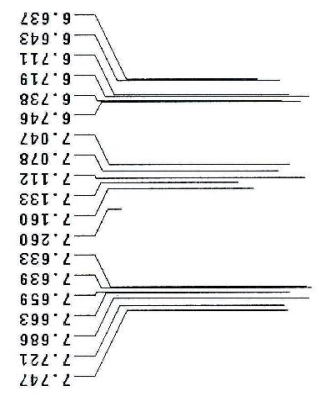

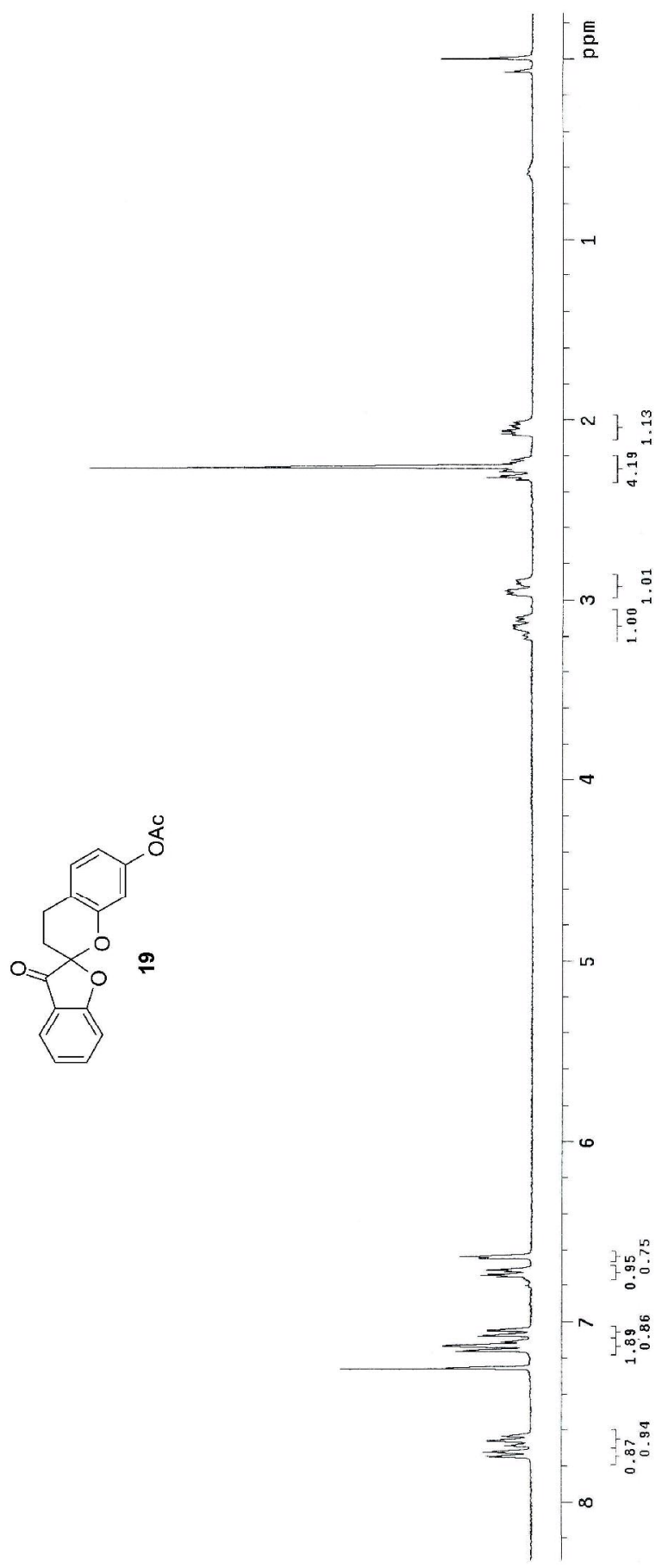



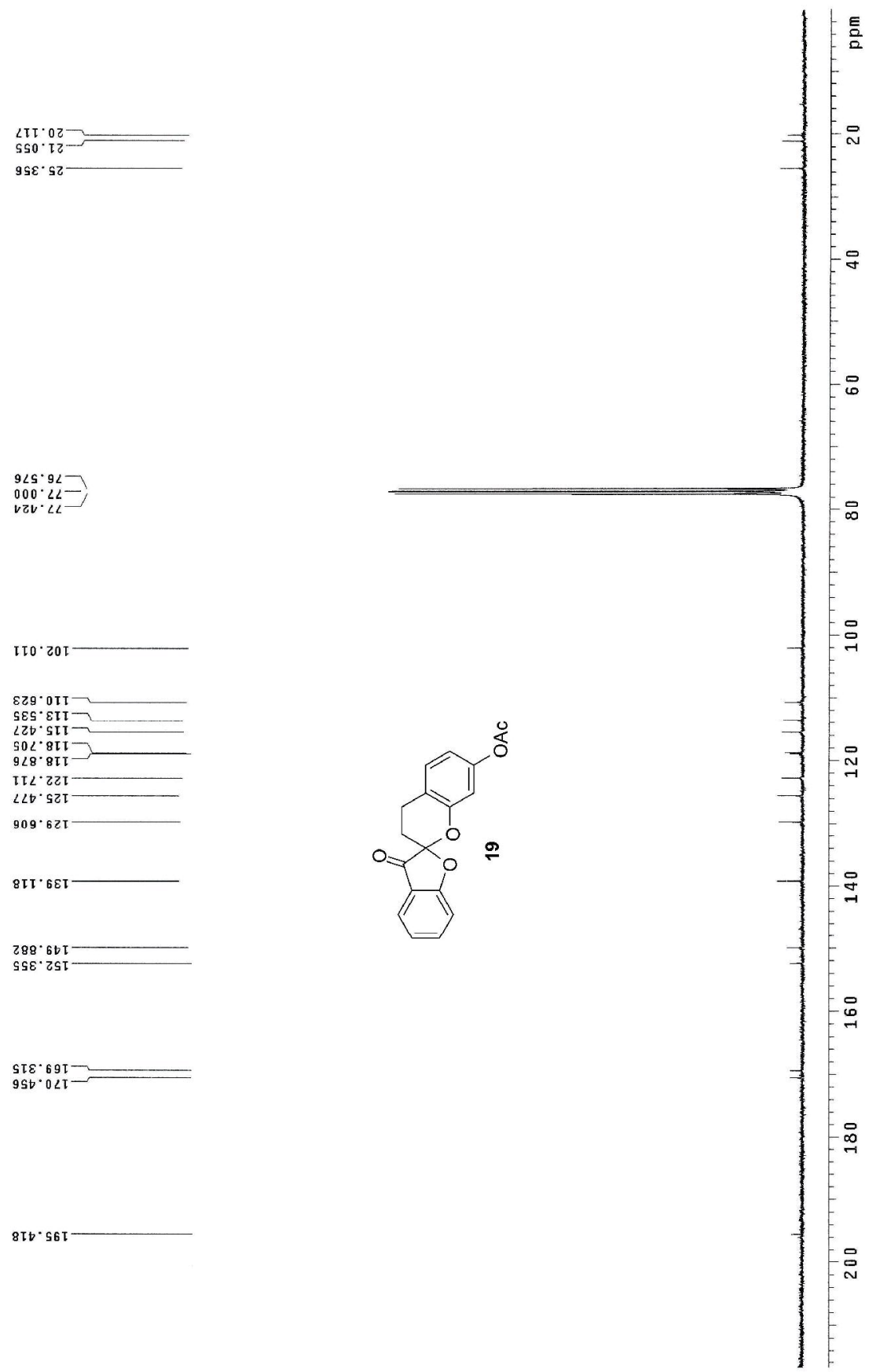

\section{References}

(1) Malkin, T.; Nierenstein, M. J. Am. Chem. Soc. 1931, 53, 239-242.

(2) Merour, J. Y.; Cossais, F. J. Heterocyclic Chem. 1991, 28, 1875-1879.

(3) Takeuchi, Y.; Watanabe, I.; Irie, K. Mari.; Hirose, Yoko.; Hirata, Kazumi.; Yamato, M.; Harayama, Takashi. Chem. Pharm. Bull. 1997, 45, 2011-2015. 